\title{
STASHING AND PARALLELIZATION PENTAGONS
}

\author{
VASCO BRATTKA \\ Faculty of Computer Science, Universität der Bundeswehr München, Germany and Department of \\ Mathematics and Applied Mathematics, University of Cape Town, South Africa \\ e-mail address: Vasco.Brattka@cca-net.de
}

\begin{abstract}
Parallelization is an algebraic operation that lifts problems to sequences in a natural way. Given a sequence as an instance of the parallelized problem, another sequence is a solution of this problem if every component is instance-wise a solution of the original problem. In the Weihrauch lattice parallelization is a closure operator that corresponds to the bang operator in linear logic. Here we introduce a dual operation that we call stashing and that also lifts problems to sequences, but such that only some component has to be an instance-wise solution. In this case the solution is stashed away in the sequence. This operation, if properly defined, induces an interior operator in the Weihrauch lattice, which corresponds to the question mark operator known from linear logic. It can also be seen as a countable version of the sum operation. We also study the action of the monoid induced by stashing and parallelization on the Weihrauch lattice, and we prove that it leads to at most five distinct degrees, which (in the maximal case) are always organized in pentagons. We also introduce another closely related interior operator in the Weihrauch lattice that replaces solutions of problems by upper Turing cones that are strong enough to compute solutions. It turns out that on parallelizable degrees this interior operator corresponds to stashing. This implies that, somewhat surprisingly, all problems which are simultaneously parallelizable and stashable have computability-theoretic characterizations. Finally, we apply all these results in order to study the recently introduced discontinuity problem, which appears as the bottom of a number of natural stashing-parallelization pentagons. The discontinuity problem is not only the stashing of several variants of the lesser limited principle of omniscience, but it also parallelizes to the non-computability problem. This supports the slogan that "non-computability is the parallelization of discontinuity". We also study the non-majorization problem as an asymmetric version of the discontinuity problem and we show that it parallelizes to the hyperimmunity problem. Finally we identify a phase transition related to the limit avoidance problem that marks a point where pentagons are taking off from the bottom of the Weihrauch lattice.
\end{abstract}

\section{INTRODUCTION}

The Weihrauch lattice has been used as a computability theoretic framework to analyze the uniform computational content of mathematical problems from many different areas of mathematics, and it can also be seen as a uniform variant of reverse mathematics (a recent survey on Weihrauch complexity can be found in [BGP21]).

The notion of a mathematical problem has a very general definition in this approach.

Key words and phrases: Weihrauch complexity, computable analysis, computability theory, closure and interior operators, linear logic.

님

(c) V. Brattka (c) Creative Commons 
Definition 1.1 (Problems). A problem is a multi-valued function $f: \subseteq X \rightrightarrows Y$ on represented spaces $X, Y$ that has a realizer.

We recall that by a realizer $F: \subseteq \mathbb{N}^{\mathbb{N}} \rightarrow \mathbb{N}^{\mathbb{N}}$ of $f$, we mean a function $F$ that satisfies $\delta_{Y} F(p) \in f \delta_{X}(p)$ for all $p \in \operatorname{dom}\left(f \delta_{X}\right)$, where $\delta_{X}: \subseteq \mathbb{N}^{\mathbb{N}} \rightarrow X$ and $\delta_{Y}: \subseteq \mathbb{N}^{\mathbb{N}} \rightarrow Y$ are the representations of $X$ and $Y$, respectively (i.e., partial surjective maps onto $X$ and $Y$, respectively). A problem is called computable if it has a computable realizer and continuous if it has a continuous realizer.

By $\langle p, q\rangle$ we denote the usual pairing function on $\mathbb{N}^{\mathbb{N}}$, defined by $\langle p, q\rangle(2 n)=p(n)$, $\langle p, q\rangle(2 n+1)=q(n)$ for all $p, q \in \mathbb{N}^{\mathbb{N}}, n \in \mathbb{N}$. Weihrauch reducibility can now be defined as follows.

Definition 1.2 (Weihrauch reducibility). Let $f: \subseteq X \rightrightarrows Y$ and $g: \subseteq W \rightrightarrows Z$ be problems. Then $f$ is called Weihrauch reducible to $g$, in symbols $f \leq_{\mathrm{W}} g$, if there are computable $H, K: \subseteq \mathbb{N}^{\mathbb{N}} \rightarrow \mathbb{N}^{\mathbb{N}}$ such that $H\langle\mathrm{id}, G K\rangle$ is a realizer of $f$ whenever $G$ is a realizer of $g$. Analogously, one says that $f$ is strongly Weihrauch reducible to $g$, in symbols $f \leq_{\mathrm{sW}} g$, if the expression $H\langle\mathrm{id}, G K\rangle$ can be replaced by $H G K$. Both versions of the reducibility have topological counterparts, where one only requires $H, K$ to be continuous and these reducibilities are denoted by $\leq_{\mathrm{W}}^{*}$ and $\leq_{\mathrm{sW}}^{*}$, respectively.

The topological version of Weihrauch reducibility has always been studied alongside the computability-theoretic version, and all four reducibilities induce a lattice structure (see [BGP21] for references).

Normally, the Weihrauch lattice refers to the lattice induced by $\leq_{\mathrm{W}}$, but here we will freely use this term also for the lattice structure induced by $\leq_{\mathrm{W}}^{*}$. If we want to be more precise, we will call the latter the topological Weihrauch lattice. The equivalence classes of problems under (strong) Weihrauch reducibility are called (strong) Weihrauch degrees.

In [BG11b, Definition 4.1] the operation of parallelization was introduced. For reasons that will become clear below, we denote the parallelization $\widehat{f}$ of a problem $f$ additionally with the non-standard notation $\Pi f$ in this article.

Definition 1.3 (Parallelization). For every problem $f: \subseteq X \rightrightarrows Y$ we define its parallelization $\Pi f: \subseteq X^{\mathbb{N}} \rightrightarrows Y^{\mathbb{N}}$ by $\operatorname{dom}(\Pi f):=\operatorname{dom}(f)^{\mathbb{N}}$ and

$$
\Pi f\left(x_{n}\right):=\left\{\left(y_{n}\right) \in Y^{\mathbb{N}}:(\forall n) y_{n} \in f\left(x_{n}\right)\right\}
$$

for all $\left(x_{n}\right) \in \operatorname{dom}(\Pi f)$. We also write $\widehat{f}:=\Pi f$ and we call a problem parallelizable (or strongly parallelizable) if $f \equiv_{\mathrm{W}} \widehat{f}$ (or $f \equiv_{\mathrm{sW}} \widehat{f}$ ) holds.

In [BG11b, Proposition 4.2] it was proved that parallelization is a closure operator in the Weihrauch lattice. This holds analogously for the topological versions of Weihrauch reducibility.

Fact 1.4 (Parallelization). $f \mapsto \Pi f$ is a closure operator with respect to the following versions of Weihrauch reducibility: $\leq_{\mathrm{W}}, \leq_{\mathrm{sW}}, \leq_{\mathrm{W}}^{*}$ and $\leq_{\mathrm{sW}}^{*}$.

We recall the definition of a closure operator and an interior operator for a preordered set. By a preordered set $(P, \leq)$ we mean a set $P$ with a relation $\leq$ on $P$ that is reflexive and transitive. The relations $\leq_{\mathrm{W}}, \leq_{\mathrm{sW}}, \leq_{\mathrm{W}}^{*}$ and $\leq_{\mathrm{sW}}^{*}$ are preorders on the set $P$ of problems $f: \subseteq \mathbb{N}^{\mathbb{N}} \rightrightarrows \mathbb{N}^{\mathbb{N}}$ on Baire space ${ }^{1}$.

\footnotetext{
${ }^{1}$ For studying the order structure it is sufficient to consider problems on Baire space as representatives of arbitrary problems. This guarantees that $P$ is actually a set.
} 
Definition 1.5 (Closure and interior operator). Let $(P, \leq)$ a preordered set with a function $C: P \rightarrow P$. Then $C$ is called a closure operator for $\leq$ if the following hold for all $x, y \in P$ :

(1) $x \leq C(x)$

(2) $x \leq y \Longrightarrow C(x) \leq C(y)$

(extensive)

(3) $C C(x) \leq C(x)$

Analogously, $C$ is called an interior operator for $\leq$ if the three conditions hold for $\geq$ in place of $\leq$.

Besides $f \mapsto \Pi f$ a number of other closure operator appeared in the study of the Weihrauch lattice [BGP21]. However, not so many interior operators have been considered yet. In this article we want to study a dual operation to parallelization that we call stashing and that can be defined as follows.

Definition 1.6 (Stashing). For every problem $f: \subseteq X \rightrightarrows Y$ we define its stashing or summation $\Sigma f: \subseteq X^{\mathbb{N}} \rightrightarrows \bar{Y}^{\mathbb{N}}$ by $\operatorname{dom}(\Sigma f):=\operatorname{dom}(f)^{\mathbb{N}}$ and

$$
\Sigma f\left(x_{n}\right):=\left\{\left(y_{n}\right) \in \bar{Y}^{\mathbb{N}}:(\exists n) y_{n} \in f\left(x_{n}\right)\right\}
$$

for all $\left(x_{n}\right) \in \operatorname{dom}(\Sigma f)$. We also write $\underset{f}{f}:=\Sigma f$ and we call a problem stashable (or strongly stashable) if $f \equiv_{\mathrm{W}} \underset{\sim}{f}$ (or $f \equiv_{\mathrm{sW}} \underset{\sim}{f}$ ) holds.

Essentially, the definition corresponds to parallelization with an existential quantifier in the place of the universal one. This means that given an instance $\left(x_{n}\right)$ for $\Sigma f$, a solution is a sequence $\left(y_{n}\right)$ such that $y_{n} \in f\left(x_{n}\right)$ for at least one $n \in \mathbb{N}$. This operation can be seen as a countable version of the sum operation + (see [BGP21]), which is the reason why we have called it summation in earlier presentations of this work. However, stashing better corresponds to the intuition of what $\Sigma f$ does.

A subtle technical point in this definition is that we use the completion $\bar{Y}$ of the space $Y$ on the output side. For a represented space $\left(Y, \delta_{Y}\right)$ the completion $\left(\bar{Y}, \delta_{\bar{Y}}\right)$ is defined by $\bar{Y}:=Y \cup\{\perp\}$ (with a distinct element $\perp \notin Y$ ) and $\delta_{\bar{Y}}: \mathbb{N}^{\mathbb{N}} \rightarrow \bar{Y}$ with

$$
\delta_{\bar{Y}}(p):=\left\{\begin{array}{ll}
\delta_{Y}(p-1) & \text { if } p-1 \in \operatorname{dom}\left(\delta_{Y}\right) \\
\perp & \text { otherwise }
\end{array},\right.
$$

where $p-1 \in \mathbb{N}^{\mathbb{N}} \cup \mathbb{N}^{*}$ is a finite or infinite sequence that is obtained as the concatenation of $p(0)-1, p(1)-1, p(2)-1, \ldots$ with the understanding that $-1=\varepsilon \in \mathbb{N}^{*}$ is the empty word. This operation of completion saw some recent surge of interest after Dzhafarov [Dzh19] used it to show that the strong version Weihrauch reducibility $\leq_{\mathrm{sW}}$ actually yields a lattice structure (here completion appeared in the definition of a suitable supremum operation). See [BG20, BG21] for further applications and a more detailed study of completion.

One reason that the completion cannot be omitted in Definition 1.6 is that it allows us to produce dummy outputs with no meaning (without the completion this might not be possible as, for instance, some represented spaces $\left(Y, \delta_{Y}\right)$ might not even have computable points). Another reason is that every partial computable problem with a completion on the output side can be extended to a total computable problem in a certain sense. In any case the completion enables us to prove the following result (see Proposition 2.2) in Section 2.

Proposition 1.7 (Stashing). $f \mapsto \Sigma f$ is an interior operator with respect to the following versions of Weihrauch reducibility: $\leq_{\mathrm{W}}, \leq_{\mathrm{sW}}, \leq_{\mathrm{W}}^{*}$ and $\leq_{\mathrm{sW}}^{*}$. 


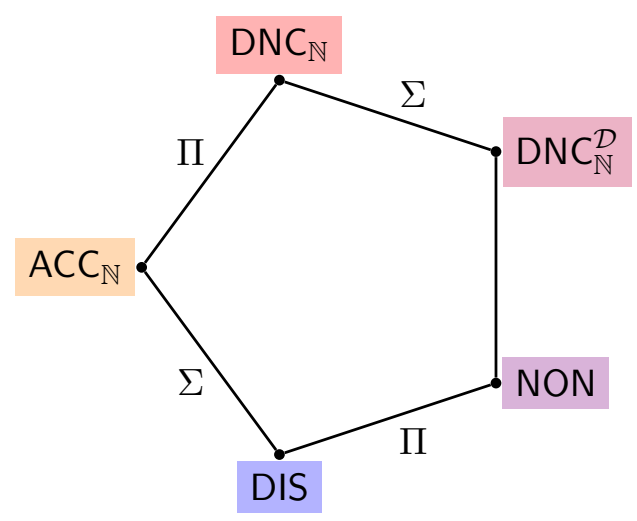

Figure 1: $A C C_{\mathbb{N}}$ pentagon in the Weihrauch lattice.

While parallelization $\Pi$ can be seen as the counterpart of the bang operator "!' in linear logic [BGP21], stashing $\Sigma$ can be seen as the counterpart of the dual why-not operator "?".

Since our lattice is now equipped with a closure operator $f \mapsto \Pi f$ and a dual interior operator $f \mapsto \Sigma f$, it is natural to ask how the monoid generated by $\{\Pi, \Sigma\}^{*}$ acts on the lattice structure? In other words, starting from an arbitrary problem $f$, what kind of problems can we generate by repeated applications of $\Pi$ and $\Sigma$ (in any order) to $f$ ? And how are these problems related with respect to the lattice structure?

In Section 3 we prove that we can generate at most five distinct problems in this way (up to equivalence) and that these five problems (in the maximal case) are always organized in a pentagon (see Proposition 3.2, Corollary 3.3).

The maximal case can actually occur and in Section 5 we study a number of specific such pentagons, in particular the one shown in the diagram in Figure 1. Here every problem in the diagram allows $\mathrm{a} \leq_{\mathrm{sW}}-$ reduction to any problem above it that is connected with a line and no other $\leq_{\mathrm{W}}^{*}$-reductions are possible (except for those that follow by transitivity). We define all the problems that occur in this diagram and some further problems that we are going to study in this article.

Definition 1.8 (Some problems). We consider the following problems for $X \subseteq \mathbb{N}$ :

(1) LPO : $\mathbb{N}^{\mathbb{N}} \rightarrow\{0,1\}, \operatorname{LPO}(p)=1: \Longleftrightarrow p=000 \ldots$

(2) $\lim : \subseteq \mathbb{N}^{\mathbb{N}} \rightarrow \mathbb{N}^{\mathbb{N}},\left\langle p_{0}, p_{1}, p_{2}, \ldots\right\rangle \mapsto \lim _{n \rightarrow \infty} p_{n}$,

(3) $\lim _{X}: \subseteq X^{\mathbb{N}} \rightarrow X,\left(x_{n}\right)_{n \in \mathbb{N}} \mapsto \lim _{n \rightarrow \infty} x_{n}$,

(4) $\mathrm{J}: \mathbb{N}^{\mathbb{N}} \rightarrow \mathbb{N}^{\mathbb{N}}, p \mapsto p^{\prime}$,

(5) $\mathrm{EC}: \mathbb{N}^{\mathbb{N}} \rightarrow 2^{\mathbb{N}}, p \mapsto \operatorname{range}(p-1)$,

(6) $C_{X}: \subseteq \mathbb{N}^{\mathbb{N}} \rightrightarrows X, p \mapsto X \backslash \operatorname{range}(p-1), \operatorname{dom}\left(C_{X}\right)=\left\{p \in \mathbb{N}^{\mathbb{N}}:|X \backslash \operatorname{range}(p-1)| \geq 1\right\}$,

(7) $\mathrm{ACC}_{X}: \subseteq \mathbb{N}^{\mathbb{N}} \rightrightarrows X, p \mapsto X \backslash \operatorname{range}(p-1)$ with $\operatorname{dom}\left(\mathrm{ACC}_{X}\right)=\left\{p \in \mathbb{N}^{\mathbb{N}}:|\operatorname{range}(p-1)| \leq 1\right\}$

(8) $\mathrm{AUC}_{X}: \subseteq \mathbb{N}^{\mathbb{N}} \rightrightarrows X, p \mapsto X \backslash \operatorname{range}(p-1)$ with $\operatorname{dom}\left(\mathrm{AUC}_{X}\right)=\left\{p \in \mathbb{N}^{\mathbb{N}}:|X \backslash \operatorname{range}(p-1)|=1\right.$ or range $\left.(p-1)=\emptyset\right\}$,

(9) $\mathrm{DNC}_{X}: \mathbb{N}^{\mathbb{N}} \rightrightarrows X^{\mathbb{N}}, p \mapsto\left\{q \in X^{\mathbb{N}}:(\forall i \in \mathbb{N}) \varphi_{i}^{p}(i) \neq q(i)\right\}$,

(10) PA : $\mathbb{N}^{\mathbb{N}} \rightrightarrows \mathbb{N}^{\mathbb{N}}, p \mapsto\left\{q \in \mathbb{N}^{\mathbb{N}}: p \ll q\right\}$,

(11) WKL $: \subseteq \operatorname{Tr} \rightrightarrows 2^{\mathbb{N}}, T \mapsto[T]$ with $\operatorname{dom}(\mathrm{WKL})=\{T: T$ infinite $\}$,

(12) NON : $\mathbb{N}^{\mathbb{N}} \rightrightarrows \mathbb{N}^{\mathbb{N}}, p \mapsto\left\{q \in \mathbb{N}^{\mathbb{N}}: q \not \mathbb{T}_{\mathrm{T}} p\right\}$,

(13) DIS : $\mathbb{N}^{\mathbb{N}} \rightrightarrows \mathbb{N}^{\mathbb{N}}, p \mapsto\left\{q \in \mathbb{N}^{\mathbb{N}}: \mathrm{U}(p) \neq q\right\}$, 


$$
\text { NRNG : } \mathbb{N}^{\mathbb{N}} \rightrightarrows 2^{\mathbb{N}}, p \mapsto\left\{A \in 2^{\mathbb{N}}: A \neq \operatorname{range}(p-1)\right\}
$$

Here LPO is also known as limited principle of omniscience and it is nothing but the characteristic function of the zero sequence. By lim we just denote the ordinary limit map with respect to the Baire space topology, where for convenience, the input sequence is encoded by $\left\langle p_{0}, p_{1}, p_{2}, \ldots\right\rangle\langle n, k\rangle:=p_{n}(k)$ where $\langle n, k\rangle:=\frac{1}{2}(n+k)(n+k+1)+k$ is the usual Cantor pairing function for $n, k \in \mathbb{N}$. By $p^{\prime}$ we denote the Turing jump of $p \in \mathbb{N}^{\mathbb{N}}$. We identify $n \in \mathbb{N}$ with the set $n=\{0, \ldots, n-1\}$. The problem EC (this name was introduced in [Wei00, Exercise 8.2]) was originally studied under the name $\mathrm{C}$ [vS89, Myl92, Bra99, Bra05, Myl06, BG11b]. Intuitively, EC translates enumerations into characteristic functions. The problem $C_{\mathbb{N}}$ is known as choice on the natural numbers and was introduced and studied in [BG11a, BdBP12]. The problems $\mathrm{ACC}_{X}$ are also known under the name $\mathrm{LLPO}_{X}$ and have been studied in [Wei92, HK14b, BHK17]. The acronym ACC stands for all-or-co-unique choice and LLPO $:=\mathrm{C}_{2}=\mathrm{ACC}_{2}=\mathrm{AUC}_{2}$ is known as lesser limited principle of omniscience. We recall that $p-1$ was defined above and $|A|$ denotes the cardinality of the set $A$. The acronym AUC stands for all-or-unique choice. This problem was studied mostly for the unit interval $X=[0,1]$ [Pau11, BGH15, KP16] and not for spaces $X \subseteq \mathbb{N}$ that we are interested in here. The acronym DNC stands for diagonally non-computable and by $\varphi^{p}$ we denote a standard Gödel numbering of the partial computable functions $\varphi_{i}^{p}: \subseteq \mathbb{N} \rightarrow \mathbb{N}$ relative to some oracle $p \in \mathbb{N}^{\mathbb{N}}$. The acronym PA stands for Peano arithmetic and by $p \ll q$ we express the fact that $q$ is of PA-degree relative to $p$, which means that $q$ computes a path through every infinite binary tree that is computable relative to $p$. The relation « was introduced by Simpson [Sim77]. By Tr we denote the set of binary trees $T \subseteq\{0,1\}^{*}$ and $[T]$ denotes the set of infinite paths of such a tree and WKL stands for Weak König's Lemma. By $\leq_{\mathrm{T}}$ we denote Turing reducibility and by $\mathrm{U}: \subseteq \mathbb{N}^{\mathbb{N}} \rightarrow \mathbb{N}^{\mathbb{N}}$ we denote some universal computable function. Such a function can be defined, for instance, by $\mathrm{U}\langle\langle i, r\rangle, p\rangle:=\varphi_{i}^{\langle r, p\rangle}$ whenever $\varphi_{i}^{\langle r, p\rangle}$ is total (and undefined otherwise). ${ }^{2}$ Here $\langle i, r\rangle:=i r$ for $i \in \mathbb{N}$ and $r \in \mathbb{N}^{\mathbb{N}}$. The problems NON and DIS are called the non-computability problem and the discontinuity problem, respectively. The problem NRNG is called range non-equality problem and is introduced here.

Nobrega and Pauly used Wadge games to characterize certain lower cones in the Weihrauch lattice [NP19]. In [Bra20] we have characterized the upper cone of the discontinuity problem by Wadge games on problems. The characterization goes as follows [Bra20, Theorem 17, Corollary 28].

Theorem 1.9 (Wadge games and the discontinuity problem). Let $f: \subseteq X \rightrightarrows Y$ be a problem. Then DIS $\leq_{\mathrm{W}} f \Longleftrightarrow$ Player I has a computable winning strategy in the Wadge game $f$.

An analogous result holds for $\leq_{\mathrm{W}}^{*}$ and (not necessarily computable) winning strategies [Bra20, Theorem 27]. We are going to use Theorem 1.9 in the proof of Proposition 5.2 that establishes the pentagon of $\mathrm{ACC}_{\mathbb{N}}$ shown in Figure 1.

Several facts are known about the parallelization of the problems summarized in Definition 1.8. These results were proved in [BG11b, Lemma 6.3, Theorem 8.2], [BdBP12,

\footnotetext{
${ }^{2}$ The universal function $U$ has been defined differently in [Bra20], but our definition is equivalent, as the function $U$ defined here also satisfies a utm- and an smn-theorem [Wei00]. In particular, the discontinuity problem DIS defined with one version of $U$ is strongly Weihrauch equivalent to the one defined with the other version of $\mathrm{U}$.
} 
Lemma 8.9] and the result $\widehat{\mathrm{ACC}_{X}} \equiv_{\mathrm{SW}} \mathrm{DNC}_{X}$ has first been proved by Higuchi and Kihara [HK14b, Proposition 81] and independently in [BHK17, Theorem 5.2]. See also the survey [BGP21].

Fact 1.10 (Parallelization of problems). $\widehat{\mathrm{LPO}} \equiv_{\mathrm{sW}} \widehat{\mathrm{C}_{\mathbb{N}}} \equiv_{\mathrm{sW}} \widehat{\lim _{X}} \equiv_{\mathrm{sW}} \lim \equiv_{\mathrm{sW}} \mathrm{J} \equiv_{\mathrm{sW}} \mathrm{EC}$, $\widehat{\mathrm{LLPO}} \equiv_{\mathrm{sW}} \widehat{\mathrm{C}_{n}} \equiv_{\mathrm{sW}} \mathrm{WKL}$, and $\widehat{\mathrm{ACC}_{X}} \equiv_{\mathrm{sW}} \mathrm{DNC}_{X}$ for $X=\mathbb{N}$ or $X \geq 2$ and $n \geq 2$.

Until recently the problems $\mathrm{ACC}_{\mathbb{N}}$ and NON were the two weakest unsolvable (mutually incomparable) natural problems in the Weihrauch lattice, and, in fact, they are the two weakest problems discussed in [BHK17]. Hence, it is a somewhat surprising coincidence that these problems appear together in the diagram in Figure 1. In fact, they are related through $\mathrm{NON} \equiv_{\mathrm{sW}} \Pi \Sigma\left(\mathrm{ACC}_{\mathbb{N}}\right)$. The discontinuity problem DIS was introduced in [Bra20] and it was proved that (under the axiom of determinacy) DIS is actually the weakest discontinuous problem with respect to the topological version of Weihrauch reducibility $\leq_{\mathrm{W}}^{*}$. Part of the above relation between $\mathrm{ACC}_{\mathbb{N}}$ and NON is that we are going to prove that DIS parallelizes to NON (see Theorem 5.4).

Theorem 1.11 (Non-computability is parallelized discontinuity). NON $\equiv_{\mathrm{sW}} \widehat{\mathrm{DIS}}$.

This result supports the slogan that "non-computability is parallelized discontinuity". In Section 5 with study a number of further pentagons with the discontinuity problem at the bottom and we show that the discontinuity problem can be obtain by stashing of several different problems.

Theorem 1.12 (Discontinuity as stashing). DIS $\equiv_{\mathrm{sW}} \underline{\mathrm{LPO}} \equiv_{\mathrm{sW}} \underline{\mathrm{LLPO}} \equiv_{\mathrm{sW}} \underline{\mathrm{ACC}_{\mathbb{N}}} \equiv_{\mathrm{sW}} \underline{\mathrm{AUC}_{n}}$ for $n \geq 2$.

In Section 5 we prove that this also leads to a number of further interesting characterizations of the discontinuity problem. Most notably we obtain a fully set-theoretic characterization (i.e., a characterization that does not refer to any computability theoretic notion) with the following result.

Proposition 1.13 (Range-non-equality problem). DIS $\equiv_{\mathrm{sW}}$ NRNG.

In Section 4 we introduce and study another interior operator in the Weihrauch lattice that replaces a problem by its upper Turing cone version.

Definition 1.14 (Upper Turing cone version). Let $f: \subseteq X \rightrightarrows Y$ be a problem. We define the upper Turing cone version $f^{\mathcal{D}}: \subseteq X \rightrightarrows \mathcal{D}$ by $\operatorname{dom}\left(f^{\mathcal{D}}\right):=\operatorname{dom}(f)$ and

$$
f^{\mathcal{D}}(x):=\left\{\operatorname{deg}_{\mathrm{T}}(q) \in \mathcal{D}:\left(\exists y \leq_{\mathrm{T}} q\right) y \in f(x)\right\} .
$$

Here $\mathcal{D}$ denotes the set of Turing degrees $\operatorname{deg}_{\mathrm{T}}(p)=\left\{q \in \mathbb{N}^{\mathbb{N}}: q \equiv_{\mathrm{T}} p\right\}$, represented by $\delta_{\mathcal{D}}: \mathbb{N}^{\mathbb{N}} \rightarrow \mathcal{D}, p \mapsto \operatorname{deg}_{\mathrm{T}}(p)$. If $\left(Y, \delta_{Y}\right)$ is a represented space, then we define

$$
y \leq_{\mathrm{T}} q: \Longleftrightarrow\left(\exists p \in \delta_{Y}^{-1}\{y\}\right) p \leq_{\mathrm{T}} q \Longleftrightarrow\left(\exists \text { computable } F: \subseteq \mathbb{N}^{\mathbb{N}} \rightarrow Y\right) F(q)=y .
$$

Hence, $y \leq_{\mathrm{T}} q$ means that $y$ has a name that can be computed from $q$ and if $Y=\mathbb{N}^{\mathbb{N}}$ then this is the usual version of Turing reducibility. This version of Turing reducibility has also been called representation reducibility [Mil04].

Besides the fact that $f \mapsto f^{\mathcal{D}}$ is an interior operator in the Weihrauch lattice, our main result in this direction shows that on parallelizable problems the two interior operator $f \mapsto \Sigma f$ and $f \mapsto f^{\mathcal{D}}$ coincide (up to equivalence). 
Proposition 1.15 (Closure under upper Turing cones). $\Sigma \widehat{f} \equiv_{\mathrm{sW}} \widehat{f}^{\mathcal{D}}$ for all problems $f$.

As a corollary of this result we obtain the following surprising consequence (see Corollary 4.6).

Corollary 1.16 (Closure under upper Turing cones). If $f$ is a problem that is parallelizable and stashable, then it is also closed under upper Turing cones, i.e., $f \equiv_{\mathrm{W}} f^{\mathcal{D}}$.

This means that all such problems are essentially of computability theoretic nature. This includes all problems that occur on the right-hand side of stashing-parallelization pentagons. The remarkable situation here is that parallelization and stashing are in some sense purely set-theoretic operations (with no mention of any computability theoretic property or notion) and yet a combination of both generates computability-theoretic problems (starting from any problem $f$ whatsoever).

In Section 6 we study the non-majorization problem NMAJ as an asymmetric version of the discontinuity problem and we investigate its pentagon. This is of interest as NMAJ parallelizes to the hyperimmunity problem HYP. In Section 7 we investigate the retraction problem $\mathrm{RET}_{X}$ that characterizes the complexity of multi-valued retractions $R: \bar{X} \rightrightarrows X$ and turns out to be equivalent to $A \cup C_{X}$ for $X \geq 2$. We also study the corresponding pentagons. Finally, in Section 8 we identify a phase transition related to the limit avoidance problem $\mathrm{NLIM}_{\mathbb{N}}$. This is the weakest problem known to us that does not stash away to the discontinuity problem.

\section{Stashing as Interior Operator}

The main purpose of this section is to show that stashing $f \mapsto \Sigma f$ is an interior operator for various versions of the Weihrauch lattice (see Proposition 2.2). For this result it is essential that the completion $\bar{Y}$ of $Y$ is used on the output side of $\Sigma f: \subseteq X^{\mathbb{N}} \rightrightarrows \bar{Y}^{\mathbb{N}}$. As a technical preparation we need the following lemma. In [BG21, Corollary 2.7] we proved that there is a computable retraction $r: \overline{\bar{Y}} \rightarrow \bar{Y}$, which is a computable map such that $\left.r\right|_{\bar{Y}}=\mathrm{id} \mathrm{d}_{\bar{Y}}$. Here we will have to use a similar property of the product space $\bar{Y}^{\mathbb{N}}$.

Lemma 2.1 (Retractions for product spaces). For every represented space $Y$ there is a computable retraction $r: \overline{\bar{Y}^{\mathbb{N}}} \rightarrow \bar{Y}^{\mathbb{N}}$, i.e., a computable $r$ with $\left.r\right|_{\bar{Y}^{\mathbb{N}}}=\mathrm{id}_{\bar{Y}^{\mathbb{N}}}$.

Proof. The space $Z:=\bar{Y}$ is represented by a total representation $\delta_{Z}=\delta_{\bar{Y}}$ and by [BG21, Corollary 2.7] there is a computable retraction $R: \bar{Z} \rightarrow Z$. We assume that $Z^{\mathbb{N}}$ is represented by the completion $\delta_{\overline{Z^{\mathbb{N}}}}$ of the usual product representation $\delta_{Z^{\mathbb{N}}}$. We claim that there is a computable map $T: \overline{Z^{\mathbb{N}}} \rightarrow \bar{Z}^{\mathbb{N}}$ with $\left.T\right|_{Z^{\mathbb{N}}}=\operatorname{id}_{Z^{\mathbb{N}}}$. We assume that $\overline{Z^{\mathbb{N}}}=Z^{\mathbb{N}} \cup\left\{\perp_{\mathbb{N}}\right\}$ and $\bar{Z}=Z \cup\{\perp\}$. The names of $\perp_{\mathbb{N}}$ with respect to $\delta_{\overline{Z^{\mathbb{N}}}}$ are exactly those names that contain only finitely many digits different from 0 (since $\delta_{Z}$ and hence $\delta_{Z^{\mathbb{N}}}$ are total). Now $T$ can be realized in a computable way by interpreting the non-zero content of a given name $p$ as a name $\left\langle p_{0}, p_{1}, p_{2}, \ldots\right\rangle$ of a point in $\bar{Z}^{\mathbb{N}}$ with respect to $\delta_{\bar{Z}^{\mathbb{N}}}$. As long as no non-zero content is available in $p$, the names $p_{i}$ are filled up by zeros. Altogether this shows that $T$ is computable and it acts as the identity with the exception that $T\left(\perp_{\mathbb{N}}\right)=(\perp, \perp, \perp, \ldots)$. Now $r: \overline{Z^{\mathbb{N}}} \rightarrow Z^{\mathbb{N}}$ with $r:=\widehat{R} \circ T$ is the desired computable retraction for $Z^{\mathbb{N}}$. 
Now we are prepared to prove that stashing is an interior operator on the (strong) Weihrauch lattice. Properties (1) and (2) in the following result are only made possible by the usage of the completion $\bar{Y}$, whereas the existence of a retraction according to Lemma 2.1 guarantees that the completion is not an obstacle for property (3). For a problem $f: \subseteq X \rightrightarrows Y$ the completion is defined by

$$
\bar{f}: \bar{X} \rightrightarrows \bar{Y}, x \mapsto \begin{cases}f(x) & \text { if } x \in \operatorname{dom}(f) \\ \perp & \text { otherwise }\end{cases}
$$

This completion was introduced and studied in [BG20, BG21] and we will use it in part (2) of the following proof.

Proposition 2.2 (Stashing). The stashing operation $f \mapsto \Sigma f$ is an interior operator for $\leq_{\mathrm{sW}}, \leq_{\mathrm{W}}, \leq_{\mathrm{W}}^{*}$ and $\leq_{\mathrm{sW}}^{*}$. That is, for all problems $f, g$ :

(1) $\Sigma f \leq_{\mathrm{sW}} f$

(2) $f \leq_{\mathrm{sW}} g \Longrightarrow \Sigma f \leq_{\mathrm{sW}} \Sigma g$,

(3) $\Sigma f \leq_{\mathrm{sW}} \Sigma \Sigma f$.

Analogous statements hold for $\leq_{\mathrm{W}}, \leq_{\mathrm{W}}^{*}$ and $\leq_{\mathrm{sW}}^{*}$.

Proof. If we prove (1) and (3) for $\leq_{\mathrm{sW}}$, then this implies the corresponding statements for $\leq_{\mathrm{sW}}^{*}, \leq_{\mathrm{W}}$ and $\leq_{\mathrm{W}}^{*}$. Only in the case of $(2)$ we explicitly need to consider the different types of reducibilities.

(1) We consider the computable projection $K: X^{\mathbb{N}} \rightarrow X,\left(x_{n}\right) \mapsto x_{0}$ and the computable function $H: Y \rightarrow \bar{Y}^{\mathbb{N}}, y \mapsto(y, \perp, \perp, \ldots)$. Here $H$ is computable, since the embedding $\iota: Y \rightarrow \bar{Y}$ is computable according to [BG20, Corollary 3.10] and the element $\perp \in \bar{Y}$ is computable too. These two functions $K, H$ witness the reduction $\Sigma f \leq_{\mathrm{sW}} f$, i.e., $\operatorname{Hf} K\left(x_{n}\right) \in$ $\Sigma f\left(x_{n}\right)$ for all $\left(x_{n}\right) \in \operatorname{dom}(\Sigma f)$. We note that the usage of the completion $\bar{Y}$ guarantees the existence of computable default outputs $\perp \in \bar{Y}$.

(2) We consider problems $f: \subseteq X \rightrightarrows Y$ and $g: \subseteq W \rightrightarrows Z$. If $f \leq_{\mathrm{sW}} g$, then there are computable $K: \subseteq X \rightrightarrows W$ and $H: \subseteq Z \rightrightarrows Y$ such that $\emptyset \neq H g K(x) \subseteq f(x)$ for all $x \in \operatorname{dom}(f)$ [BGP21, Proposition 3.2]. Then the completion $\bar{H}: \bar{Z} \rightrightarrows \bar{Y}$ is computable by [BG20, Proposition 4.9] and so are the parallelizations $\widehat{\bar{H}}: \bar{Z}^{\mathbb{N}} \rightrightarrows \bar{Y}^{\mathbb{N}}$ and $\widehat{K}: \subseteq X^{\mathbb{N}} \rightrightarrows W^{\mathbb{N}}$. We obtain

$$
\begin{aligned}
\emptyset & \neq \widehat{\bar{H}} \circ \Sigma g \circ \widehat{K}\left(\left(x_{n}\right)_{n}\right) \\
& =\widehat{\bar{H}}\left\{\left(z_{n}\right)_{n} \in \bar{Z}^{\mathbb{N}}:(\exists n) z_{n} \in g K\left(x_{n}\right)\right\} \\
& =\left\{\left(y_{n}\right) \in \bar{Y}^{\mathbb{N}}:(\exists n) y_{n} \in H g K\left(\left(x_{n}\right)_{n}\right)\right\} \subseteq \Sigma f\left(\left(x_{n}\right)_{n}\right)
\end{aligned}
$$

for all $\left(x_{n}\right)_{n} \in \operatorname{dom}(\Sigma f)$. We note that the totality of the completion $\bar{H}$ guarantees that also those components of $\left(z_{n}\right)_{n} \in \Sigma g \circ \widehat{K}\left(\left(x_{n}\right)_{n}\right) \subseteq \bar{Z}^{\mathbb{N}}$ are processed, which are not in the domain of $H$. Altogether, this proves $\Sigma f \leq_{\mathrm{sW}} \Sigma g$.

Let now $f \leq{ }_{\mathrm{W}} g$ hold with computable $K: \subseteq X \rightrightarrows \mathbb{N}^{\mathbb{N}} \times W$ and $H: \subseteq \mathbb{N}^{\mathbb{N}} \times Z \rightrightarrows Y$ according to [BP18, Lemma 2.5], i.e., $\emptyset \neq H \circ\left(\operatorname{id}_{\mathbb{N}^{N}} \times g\right) \circ K(x) \subseteq f(x)$ for all $x \in \operatorname{dom}(f)$. Then, again, the completion $\bar{H}: \overline{\mathbb{N}^{\mathbb{N}} \times Z} \rightrightarrows \bar{Y}$ is computable. By [BG20, Proposition 3.8, Corollary 3.10] there is a computable $\iota: \mathbb{N}^{\mathbb{N}} \times \bar{Z} \rightarrow \overline{\mathbb{N}^{\mathbb{N}} \times Z}$ with $\iota(p, z)=(p, z)$ for all $(p, z) \in \mathbb{N}^{\mathbb{N}} \times Z$. Then $\left(\widehat{\bar{H} \circ \iota)}:\left(\mathbb{N}^{\mathbb{N}} \times \bar{Z}\right)^{\mathbb{N}} \rightrightarrows \bar{Y}^{\mathbb{N}}\right.$ can also be considered as a problem of type $H^{\prime}:\left(\mathbb{N}^{\mathbb{N}}\right)^{\mathbb{N}} \times \bar{Z}^{\mathbb{N}} \rightrightarrows \bar{Y}^{\mathbb{N}}$ 
and $\widehat{K}$ can be seen as a problem of type $\widehat{K}: \subseteq X^{\mathbb{N}} \rightrightarrows\left(\mathbb{N}^{\mathbb{N}}\right)^{\mathbb{N}} \times W^{\mathbb{N}}$. As above we obtain

$$
\emptyset \neq H^{\prime} \circ\left(\operatorname{id}_{\left(\mathbb{N}^{\mathbb{N}}\right)^{\mathbb{N}}} \times \Sigma g\right) \circ \widehat{K}\left(\left(x_{n}\right)_{n}\right) \subseteq \Sigma f\left(\left(x_{n}\right)_{n}\right),
$$

for all $\left(x_{n}\right)_{n} \in \operatorname{dom}(\Sigma f)$, i.e., $\Sigma f \leq_{\mathrm{W}} \Sigma g$. The statements for $\leq_{\mathrm{sW}}^{*}$ and $\leq_{\mathrm{W}}^{*}$ can be proved analogously with continuous $K, H$. We note that by [BG20, Proposition 4.9] $\bar{H}$ is continuous, if $H$ is so.

(3) We consider $\Sigma \Sigma f: \subseteq\left(X^{\mathbb{N}}\right)^{\mathbb{N}} \rightrightarrows{\overline{Y^{\mathbb{N}}}}^{\mathbb{N}}$. By Lemma 2.1 there is a computable retraction $r: \overline{\bar{Y}^{\mathbb{N}}} \rightarrow \bar{Y}^{\mathbb{N}}$. For every represented space $X$ the map

$$
s_{X}:\left(X^{\mathbb{N}}\right)^{\mathbb{N}} \rightarrow X^{\mathbb{N}},\left(\left(x_{n, k}\right)_{k}\right)_{n} \mapsto\left(x_{n, k}\right)_{\langle n, k\rangle}
$$

that interleaves a double sequence in a single sequence, is a computable isomorphism, i.e., it is bijective and computable and its inverse is computable too. We now consider the computable functions $K:=s_{X}^{-1}$ and $H:=s_{Y} \circ \widehat{r}: \bar{Y}^{\mathbb{N}^{\mathbb{N}}} \rightarrow \bar{Y}^{\mathbb{N}}$. Then we obtain

$$
\begin{aligned}
\emptyset & \neq H \circ \Sigma \Sigma f \circ K\left(\left(x_{n, k}\right)_{\langle n, k\rangle}\right) \\
& =s_{Y} \circ \widehat{r}\left\{\left(z_{n}\right)_{n} \in{\overline{\bar{Y}^{\mathbb{N}}}}^{\mathbb{N}}:(\exists n) z_{n} \in \Sigma f\left(\left(x_{n, k}\right)_{k}\right)\right\} \\
& =s_{Y}\left\{\left(\left(y_{n, k}\right)_{k}\right)_{n} \in\left(\bar{Y}^{\mathbb{N}}\right)^{\mathbb{N}}:(\exists n)(\exists k) y_{n, k} \in f\left(x_{n, k}\right)\right\} \\
& =\left\{\left(y_{n, k}\right)_{\langle n, k\rangle} \in \bar{Y}^{\mathbb{N}}:(\exists\langle n, k\rangle) y_{n, k} \in f\left(x_{n, k}\right)\right\} \\
& =\Sigma f\left(\left(x_{n, k}\right)_{\langle n, k\rangle}\right) .
\end{aligned}
$$

This proves $\Sigma f \leq_{\mathrm{sW}} \Sigma \Sigma f$.

We can conclude from property (2) in Proposition 2.2 that stashing is, in particular, invariant under (strong) Weihrauch reducibility and can hence be seen as an operation on (strong) Weihrauch degrees.

Corollary 2.3. Stashing can be extended to an operation on (strong) Weihrauch degrees.

\section{The Stashing-Parallelization Monoid}

We adopt the convention that we denote the parallelization of a problem $f$ by $\widehat{f}$ and the stashing by $f$ when we deal with single applications of these operators. However, for iterated applications it is useful to use the notation $\Pi f$ and $\Sigma f$ instead.

The closure and interior operators $\Pi$ and $\Sigma$ generate a monoid $\{\Pi, \Sigma\}^{*}$ under composition and we want to study the action of this monoid on the Weihrauch lattice. To this end, it is worth spelling out the problems $\Sigma \Pi f$ and $\Pi \Sigma f$ explicitly:

(1) $\Sigma \Pi f: \subseteq X^{\mathbb{N} \times \mathbb{N}} \rightrightarrows{\overline{Y^{\mathbb{N}}}}^{\mathbb{N}},\left(x_{n, k}\right) \mapsto\left\{\left(y_{n, k}\right):(\exists n)(\forall k) y_{n, k} \in f\left(x_{n, k}\right)\right\}$,

(2) $\Pi \Sigma f: \subseteq X^{\mathbb{N} \times \mathbb{N}} \rightrightarrows \bar{Y}^{\mathbb{N} \times \mathbb{N}},\left(x_{n, k}\right) \mapsto\left\{\left(y_{n, k}\right):(\forall n)(\exists k) y_{n, k} \in f\left(x_{n, k}\right)\right\}$.

We can see that stashing corresponds to a usage of an existential quantifier whereas parallelization corresponds to a usage of a universal quantifier in a certain sense. Hence $\Sigma \Pi$ and $\Pi \Sigma$ correspond to applications of these quantifiers in different order.

There is a subtle technical point here: we have to deal with the spaces $\bar{Y}^{\mathbb{N}}$ and $\overline{Y^{\mathbb{N}}}$, which are not computably isomorphic. We recall that $\bar{Y}^{\mathbb{N}}=(Y \cup\{\perp\})^{\mathbb{N}}$, whereas $\overline{Y^{\mathbb{N}}}=Y^{\mathbb{N}} \cup\left\{\perp_{\mathbb{N}}\right\}$. Hence, formally there is no subset relation between these two sets. In order to make the 
latter a subset of the former, we can choose $\perp_{\mathbb{N}}:=(\perp, \perp, \ldots)$, as implicitly done in the proof of Lemma 2.1. In this sense the double sequence notation $\left(y_{n, k}\right)$ in the description of $\Sigma \Pi f$ should be understood.

Besides the retraction $r$ from Lemma 2.1 we also need the maps $s, t$ that exist according to the following lemma. Intuitively speaking, $s$ maps every sequence that contains a $\perp$ to $\perp_{\mathbb{N}}$ and $t$ maps $\perp_{\mathbb{N}}$ to some sequence that contains a $\perp$ (which one it is, might depend on the given name of $\perp_{\mathbb{N}}$ ).

Lemma 3.1 (Completion of product spaces). For every represented space $Y$ there are computable $s: \bar{Y}^{\mathbb{N}} \rightarrow \overline{Y^{\mathbb{N}}}$ and $t: \overline{Y^{\mathbb{N}}} \rightrightarrows \bar{Y}^{\mathbb{N}}$ such that $\left.s\right|_{Y^{\mathbb{N}}}=\left.t\right|_{Y^{\mathbb{N}}}=\operatorname{id}_{Y^{\mathbb{N}}}$.

Proof. A suitable computable map $s: \bar{Y}^{\mathbb{N}} \rightarrow \overline{Y^{\mathbb{N}}}$ with $\left.s\right|_{Y^{\mathbb{N}}}=\left.\mathrm{id}\right|_{Y^{\mathbb{N}}}$ is realized by a computable $F: \mathbb{N}^{\mathbb{N}} \rightarrow \mathbb{N}^{\mathbb{N}}$ with the property that $F\left\langle p_{0}, p_{1}, p_{2}, \ldots\right\rangle-1=\left\langle q_{0}, q_{1}, q_{2}, \ldots\right\rangle$ with $q_{i}=p_{i}-1$ for all $p_{i}$ with $p_{i}-1 \in \mathbb{N}^{\mathbb{N}}$. This can be achieved by copying the non-zero content of $p_{i}$ subtracted by 1 into the $q_{i}$, where the resulting sequence $\left\langle q_{0}, q_{1}, q_{2}, \ldots\right\rangle$ is filled up by zeros, whenever necessary (i.e., whenever no non-zero content is available for some $p_{i}$ then the entire output is filled up only with zeros as long as no non-zero content appears). That is, if one of the $p_{i}$ is a name of $\perp \in \bar{Y}$ (either because it has only finitely many digits different from zero or because $\left.p_{i}-1 \notin \operatorname{dom}\left(\delta_{Y}\right)\right)$, then $F\left\langle p_{0}, p_{1}, \ldots\right\rangle$ is a name of $\perp_{\mathbb{N}} \in \overline{Y^{\mathbb{N}}}$. In this way, $F$ realizes the identity on $Y^{\mathbb{N}}$.

For the second part of the statement, we note that $\bar{Y}^{\mathbb{N}}$ has a precomplete and total representation by [BG20, Proposition 3.8] and hence we can extend the parallelization of the computable embedding $Y \hookrightarrow \bar{Y}$ to a computable problem $t: \overline{Y^{\mathbb{N}}} \rightrightarrows \bar{Y}^{\mathbb{N}}$ with $\left.t\right|_{Y^{\mathbb{N}}}=\mathrm{id}_{Y^{\mathbb{N}}}$ by [BG21, Proposition 2.6].

Our core observation on the action of the monoid $\{\Pi, \Sigma\}^{*}$ on the (strong) Weihrauch lattice is captured by the following result.

Proposition 3.2 (Action of the stashing-parallelization monoid). For every problem $f$ we obtain:

(1) $\Pi \Sigma f \leq_{\mathrm{sW}} \Sigma \Pi f$,

(2) $\Pi \Sigma \Pi f \equiv_{\mathrm{sW}} \Sigma \Pi f$

(3) $\Sigma \Pi \Sigma f \equiv_{\mathrm{sW}} \Pi \Sigma f$.

Proof. (1) Given an instance $\left(x_{n, k}\right)$ of $\Pi \Sigma f$, we just swap $n$ - with $k$-positions in $\left(x_{n, k}\right)$, then we apply $\Sigma \Pi f$ to the result, then we use the parallelization of the problem $t: \overline{Y^{\mathbb{N}}} \rightrightarrows \bar{Y}^{\mathbb{N}}$ from Lemma 3.1 in order to convert the output of $\Sigma \Pi f$ from $\left(\overline{Y^{\mathbb{N}}}\right)^{\mathbb{N}}$ into a double sequence in $\left(\bar{Y}^{\mathbb{N}}\right)^{\mathbb{N}}$ and then we swap the $n$ - and $k$-positions again to obtain a result in $\Pi \Sigma f\left(x_{n, k}\right)$, which is correct because

$$
(\exists k)(\forall n) y_{n, k} \in f\left(x_{n, k}\right) \Longrightarrow(\forall n)(\exists k) y_{n, k} \in f\left(x_{n, k}\right) .
$$

(2) Since $\Pi$ is a closure operator we have $\Sigma \Pi f \leq_{\mathrm{sW}} \Pi \Sigma \Pi f$ and $\Pi \Pi f \leq_{\mathrm{sW}} \Pi f$, and by (1) and monotonicity of $\Sigma$ we obtain $\Pi \Sigma \Pi f \leq_{\mathrm{sW}} \Sigma \Pi \Pi f \leq_{\mathrm{sW}} \Sigma \Pi f$.

(3) Since $\Sigma$ is an interior operator we have $\Sigma \Pi \Sigma f \leq_{\mathrm{sW}} \Pi \Sigma f$ and $\Sigma f \leq \Sigma \Sigma f$, and by (1) and monotonicity of $\Pi$ we obtain $\Pi \Sigma f \leq_{\mathrm{sW}} \Pi \Sigma \Sigma f \leq_{\mathrm{sW}} \Sigma \Pi \Sigma f$.

That is the action of the stashing-parallelization monoid $\{\Pi, \Sigma\}^{*}$ on a problem $f$ in the (strong) Weihrauch lattice leads to at most five distinct degrees $\{f, \Pi f, \Sigma f, \Sigma \Pi f, \Pi \Sigma f\}$, 


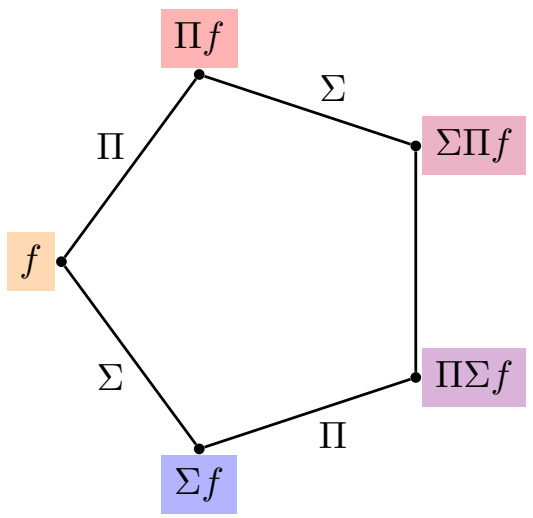

Figure 2: Parallelization-stashing pentagon in the Weihrauch lattice.

which are arranged in a pentagon, see the diagram in Figure 2. Every line in the diagram indicates a $\leq_{\mathrm{sW}}$-reduction in the upwards direction.

Of course, if a problem $f$ is parallelizable and stashable (such as any problem of the form $f=\Sigma \Pi g$ is), then the pentagon reduces to a single degree. Other smaller sizes than five can be realized too and, as we will see, also the maximal size of five can be realized. In any case, the stashing-parallelization pentagon can be seen as the trace of $f$ under $\{\Sigma, \Pi\}^{*}$ that reveals some information about the underlying problem $f$. It follows from Proposition 3.2 and the fact that $\Pi$ and $\Sigma$ are closure and interior operators, respectively, that for a problem $f$ with a full pentagon of size five, $f$ is incomparable with the opposite problems $\Sigma \Pi f$ and $\Pi \Sigma f$.

Corollary 3.3 (Full pentagons). For every problem $f$ we obtain:

(1) $f \leq_{\mathrm{sW}} \Sigma \Pi f \Longleftrightarrow \Pi f \equiv_{\mathrm{sW}} \Sigma \Pi f$,

(2) $\Sigma \Pi f \leq_{\mathrm{sW}} f \Longleftrightarrow \Sigma f \equiv_{\mathrm{sW}} \Sigma \Pi f$,

(3) $f \leq_{\mathrm{sW}} \Pi \Sigma f \Longleftrightarrow \Pi f \equiv_{\mathrm{sW}} \Pi \Sigma f$,

(4) $\Pi \Sigma f \leq_{\mathrm{sW}} f \Longleftrightarrow \Sigma f \equiv_{\mathrm{sW}} \Pi \Sigma f$.

Analogous statements hold for the reductions $\leq_{\mathrm{W}}, \leq_{\mathrm{sW}}^{*}, \leq_{\mathrm{W}}^{*}$.

There are many interesting questions regarding the interaction of parallelization and stashing. For instance, we will see later in Corollary 7.11 that the map $f \mapsto(\Sigma f, \Pi f)$ is not injective on Weihrauch degrees, i.e., problems are not characterized by their respective pentagons. However, these pentagons still reveal some interesting information in many cases, as we will see.

\section{The Upper Turing Cone Operator}

The main purpose of this section is to prove that the upper Turing cone operator $f \mapsto f^{\mathcal{D}}$ is an interior operator on the (strong) Weihrauch lattice that coincides with the interior operator $f \mapsto \Sigma f$, restricted to (strongly) parallelizable problems.

In the following it is useful to have a simplified version of $\Sigma \Pi f$, where we replace double sequences on the input side by ordinary sequences. For this purpose we consider the injection $I_{X}: X \hookrightarrow X^{\mathbb{N}}, x \mapsto(x, x, x, \ldots)$ for every represented space $X$.

Lemma 4.1. $\Sigma \Pi f \equiv_{\mathrm{sW}}(\Sigma \Pi f) \circ I_{X^{\mathbb{N}}}$ for every problem $f: \subseteq X \rightrightarrows Y$. 
Proof. Since $I_{X^{\mathbb{N}}}$ is computable, it is clear that $(\Sigma \Pi f) \circ I_{X^{\mathbb{N}}} \leq_{\mathrm{sW}} \Sigma \Pi f$ holds. On the other hand, given an instance $\left(\left(x_{n, k}\right)_{k}\right)_{n} \in X^{\mathbb{N}^{2}}$ of $\Sigma \Pi f$, we can compute the interleaved sequence $\left(x_{n, k}\right)_{\langle n, k\rangle} \in X^{\mathbb{N}}$ and then apply $I_{X^{\mathbb{N}}}$ followed by $\Sigma \Pi f$. This yields a sequence $\left(\left(y_{m,\langle n, k\rangle}\right)_{\langle n, k\rangle}\right)_{m} \in \bar{Y}^{\mathbb{N}} \mathbb{N}^{\mathbb{N}}$ that satisfies the property $(\exists m)(\forall\langle n, k\rangle) y_{m,\langle n, k\rangle} \in f\left(x_{n, k}\right)$. Using the parallelization of the problem $t: \overline{Y^{\mathbb{N}}} \rightrightarrows \bar{Y}^{\mathbb{N}}$ from Lemma 3.1 we can convert this into a sequence in $\bar{Y}^{\mathbb{N}^{2}}$ and then extract a diagonal sequence $\left(\left(y_{n,\langle n, k\rangle}\right)_{\langle n, k\rangle}\right)_{n} \in \bar{Y}^{\mathbb{N}^{2}}$ from it. This sequence can be converted back to ${\overline{Y^{\mathbb{N}}}}^{\mathbb{N}}$ by the parallelization of the function $s: \bar{Y}^{\mathbb{N}} \rightarrow \overline{Y^{\mathbb{N}}}$ from Lemma 3.1 and we claim that the result is a solution of $\Sigma \Pi f\left(\left(\left(x_{n, k}\right)_{k}\right)_{n}\right)$. This is because

$$
(\exists m)(\forall\langle n, k\rangle) y_{m,\langle n, k\rangle} \in f\left(x_{n, k}\right) \Longrightarrow(\exists n)(\forall k) y_{n,\langle n, k\rangle} \in f\left(x_{n, k}\right) .
$$

The exact relation between the upper Turing cone operator and stashing is captured in the following result, again with the help of the injection $I_{X}$.

Proposition 4.2 (Upper Turing cone operator). $f^{\mathcal{D}} \equiv_{\mathrm{sW}} \Sigma f \circ I_{X}$ holds for every problem $f: \subseteq X \rightrightarrows Y$. In particular, $f^{\mathcal{D}} \leq_{\mathrm{sW}} \Sigma f$.

Proof. We consider the represented spaces $\left(X, \delta_{X}\right)$ and $\left(Y, \delta_{Y}\right)$. We claim that every realizer $F: \subseteq \mathbb{N}^{\mathbb{N}} \rightarrow \mathbb{N}^{\mathbb{N}}$ of $\Sigma f \circ I_{X}$ is also a realizer of $f^{\mathcal{D}}$, which proves $f^{\mathcal{D}} \leq_{\mathrm{sW}} \Sigma f \circ I_{X}$. To this end, let $F$ be such a realizer and $x:=\delta_{X}(p) \in \operatorname{dom}\left(f^{\mathcal{D}}\right)=\operatorname{dom}(f)$. Let $\left(y_{n}\right):=\delta_{\bar{Y}^{\mathbb{N}}} F(p) \in$ $\Sigma f(x, x, \ldots)$. Let $\left\langle q_{0}, q_{1}, \ldots\right\rangle:=F(p)$ and let $G: \subseteq \mathbb{N}^{\mathbb{N}} \rightarrow \mathbb{N}^{\mathbb{N}}$ be a computable realizer of the partial inverse $\iota^{-1}: \subseteq \bar{Y} \rightarrow Y$ of the embedding $\iota: Y \rightarrow \bar{Y}$, which is computable according to [BG20, Corollary 3.10]. Then there is some $n \in \mathbb{N}$ with $y_{n}=\delta_{Y} G\left(q_{n}\right)=\delta_{\bar{Y}}\left(q_{n}\right) \in f \delta_{X}(p)=$ $f(x)$ and $y_{n} \leq_{\mathrm{T}} G\left(q_{n}\right) \leq_{\mathrm{T}} F(p)$. This proves the claim.

For the reverse reduction $\Sigma f \circ I_{X} \leq_{\mathrm{sW}} f^{\mathcal{D}}$ we first note that $\delta_{\bar{Y}}$ is a precomplete representation by [BG20, Proposition 3.8]. Hence there is a total computable $r: \mathbb{N} \rightarrow \mathbb{N}$ such that for all $q \in \mathbb{N}^{\mathbb{N}}, n \in \mathbb{N}$ the function $\varphi_{r(n)}^{q}$ is always total and if $\varphi_{n}^{q}$ is total and $\varphi_{n}^{q} \in \operatorname{dom}\left(\delta_{Y}\right)$, then $\delta_{\bar{Y}}\left(\varphi_{r(n)}^{q}\right)=\delta_{Y}\left(\varphi_{n}^{q}\right)$. Intuitively, the programme with code $r(n)$ works as the programme $n$, but it adds 1 to all output results and fills up the output with dummy symbols 0 in appropriate positions as long as no other better information becomes available. Now we consider the computable function $H: \mathbb{N}^{\mathbb{N}} \rightarrow \mathbb{N}^{\mathbb{N}}$ with $H(q):=\left\langle\varphi_{r(0)}^{q}, \varphi_{r(1)}^{q}, \ldots\right\rangle$. We claim that $H F$ is a realizer of $\Sigma f \circ I_{X}$ for every realizer $F$ of $f^{\mathcal{D}}$. To this end, let $F$ be such a realizer and $x:=\delta_{X}(p) \in \operatorname{dom}\left(\Sigma f \circ I_{X}\right)=\operatorname{dom}(f)$. Then there exists some $y \leq_{\mathrm{T}} F(p)$ with $y \in f(x)$. Hence, there is some $n \in \mathbb{N}$ such that $y=\delta_{Y}\left(\varphi_{n}^{F(p)}\right)=\delta_{\bar{Y}}\left(\varphi_{r(n)}^{F(p)}\right)$. This implies that $\delta_{\bar{Y}^{\mathbb{N}}} H F(p) \in \Sigma f \circ I_{X}(x)$, which completes the proof.

We note that the main idea of the proof, namely to compute on all Gödel numbers in parallel, can only be realized because stashing uses a completion $\bar{Y}$ of the space $Y$ on the output side.

By a combination of Propositions 4.2 and 3.2 with Lemma 4.1 we obtain the following corollary.

Corollary 4.3 (Upper Turing cone operator) $(\Pi f)^{\mathcal{D}} \equiv_{\mathrm{sW}} \Sigma \Pi f$ and $(\Pi \Sigma f)^{\mathcal{D}} \equiv_{\mathrm{sW}} \Pi \Sigma f$ for every problem $f$.

This means that both problems on the right-hand side of the diagram in Figure 2 can be seen as upper Turing cone versions and hence as computability-theoretic problems. We 
emphasize that Turing cones appear here out of a purely topological context without any computability theory being involved. This is because Corollary 4.3 is also correct when the computability-theoretic Weihrauch reducibility is replaced by its topological counterpart.

We mention in passing that the upper Turing cone operator is an interior operator on the Weihrauch lattice.

Proposition 4.4 (Upper Turing cone operator as interior operator). The operation $f \mapsto f^{\mathcal{D}}$ is an interior operator on the (strong) Weihrauch lattice. That is, for all problems $f, g$ we have:

(1) $f^{\mathcal{D}} \leq_{\mathrm{sW}} f$,

(2) $f \leq_{\mathrm{sW}} g \Longrightarrow f^{\mathcal{D}} \leq_{\mathrm{sW}} g^{\mathcal{D}}$,

(3) $f^{\mathcal{D}} \leq_{\mathrm{sW}} f^{\mathcal{D D}}$.

Analogous statements hold for $\leq_{\mathrm{W}}, \leq_{\mathrm{W}}^{*}$ and $\leq_{\mathrm{sW}}^{*}$.

Proof. We consider problems $f: \subseteq X \rightrightarrows Y$ and $g: \subseteq W \rightrightarrows Z$.

(1) This follows from $f^{\mathcal{D}} \leq_{\mathrm{sW}} \Sigma f \leq_{\mathrm{sW}} f$, which holds by Propositions 4.2 and 2.2 .

(2) Let $f \leq_{\mathrm{W}} g$ hold via computable $H, K: \subseteq \mathbb{N}^{\mathbb{N}} \rightarrow \mathbb{N}^{\mathbb{N}}$, i.e., $H\langle\mathrm{id}, G K\rangle$ is a realizer of $f$ whenever $G$ is a realizer of $g$. We claim that $f^{\mathcal{D}} \leq_{\mathrm{W}} g^{\mathcal{D}}$ holds via id, $K$. Let $p$ be a name of some input $x \in \operatorname{dom}(f)$. Then $K(p)$ is a name of a point $w \in \operatorname{dom}(g)$ and any name $q$ of a point in $g(w)$ yields a name $H\langle p, q\rangle$ of a point in $f(x)$, since there is a realizer $G$ of $g$ with $G K(p)=q$. Let now $G$ be a realizer of $g^{\mathcal{D}}$. Then there is a name $q$ of a point in $g(w)$ such that $q \leq_{\mathrm{T}} G K(p)$. Hence $H\langle p, q\rangle \leq_{\mathrm{T}}\langle p, G K(p)\rangle$. This shows that $\langle\mathrm{id}, G K\rangle$ is a realizer of $f^{\mathcal{D}}$ whenever $G$ is a realizer of $g^{\mathcal{D}}$ and hence $f^{\mathcal{D}} \leq_{\mathrm{W}} g^{\mathcal{D}}$. The statement for $\leq_{\mathrm{sW}}$ can be proved analogously. In the topological cases we have to work with continuous $H, K$. Then $H$ is computable relative to some $r \in \mathbb{N}^{\mathbb{N}}$ and we obtain as above $H\langle p, q\rangle_{\mathrm{T}_{\mathrm{T}}}\langle r, p, G K(p)\rangle$. Hence $f^{\mathcal{D}} \leq_{\mathrm{W}}^{*} g^{\mathcal{D}}$ holds via continuous $H^{\prime}, K$, where $H^{\prime}\langle p, q\rangle:=\langle r, p, q\rangle$.

(3) We have even $f^{\mathcal{D D}}=f^{\mathcal{D}}$ by transitivity of Turing reducibility.

By Proposition 3.2 stashing extends to an interior operator on parallelizable Weihrauch degrees and by Corollary 4.3 the upper Turing cone operator coincides on those degrees with stashing.

Corollary 4.5. $f \mapsto \Sigma f$ and $f \mapsto f^{\mathcal{D}}$ are identical interior operators restricted to (strongly) parallelizable (strong) Weihrauch degrees.

We note that $f \mapsto \Sigma f$ and $f \mapsto f^{\mathcal{D}}$ are not identical on arbitrary Weihrauch degrees. The problem $f^{\mathcal{D}}$ is always computable when $f$ has only computable solutions. For instance, LPO $^{\mathcal{D}}$ is computable, while this is not the case for $\Sigma($ LPO) (see Proposition 5.10).

We can also formulate this result such that problems which are simultaneously stashable and parallelizable are automatically closed under applying the upper Turing cone operator.

Corollary 4.6. For every problem $f$ the following conditions are equivalent to each other: (1) $\Sigma f \equiv_{\mathrm{sW}} f$ and $\Pi f \equiv_{\mathrm{sW}} f$,

(2) $f^{\mathcal{D}} \equiv_{\mathrm{sW}} f$ and $\Pi f \equiv_{\mathrm{sW}} f$.

An analogous property holds with $\equiv_{\mathrm{W}}$ instead of $\equiv_{\mathrm{sW}}$.

It follows from Corollary 4.3 that problems $g$ that are simultaneously parallelizable and stashable can only occur in certain regions of the Weihrauch lattice. For one, every problem with the set of Turing degrees as target set is densely realized by [BHK17, Corollary 4.9], which means that a realizer of such a problem can produce outputs that start with arbitrary 
prefixes. This in turn implies by [BP18, Proposition 6.3] that any problem with discrete output below it has to be computable. We formulate this as a corollary.

Corollary 4.7 (Parallelizable and stashable problems). Let $f: \subseteq X \rightrightarrows \mathbb{N}$ be a problem and let $g$ be a problem that is parallelizable and stashable. If $f \leq_{\mathrm{W}} g$ holds, then $f$ is computable.

One of the weakest problems with discrete output that is discontinuous is $\mathrm{ACC}_{\mathbb{N}}$, the allor-co-unique choice problem on $\mathbb{N}$. This problem was studied in [BHK17] and an equivalent problem was investigated earlier under the name LLPO $_{X}$ [Wei92, HK14a] (and under the name $\mathrm{LLPO}_{\infty}\left[\mathrm{Myl06}\right.$, Definition 16] in the case of $\mathrm{ACC}_{\mathbb{N}}$ ). Intuitively speaking, $\mathrm{ACC}_{\mathbb{N}}$ is the problem that given a list of natural numbers which is either empty or contains exactly one number, one has to produce a number which is not in the list. For $f=\mathrm{ACC}_{\mathbb{N}}$ we can also phrase Corollary 4.7 as follows.

Corollary 4.8 (The cone of all-or-co-unique choice). If $\mathrm{ACC}_{\mathbb{N}} \leq_{\mathrm{W}} g$ holds for some problem $g$, then $g$ cannot be simultaneously parallelizable and stashable.

Hence, in a certain sense, problems that are parallelizable and stashable at the same time are rare, even rarer than this result suggests. Namely, $A_{C} C_{\mathbb{N}}$ is not the weakest discontinuous problem with discrete output. Mylatz has proved that there are also discontinuous problems of type $f: \subseteq \mathbb{N}^{\mathbb{N}} \rightrightarrows \mathbb{N}$ with $f<_{\mathrm{W}} \mathrm{ACC}_{\mathbb{N}}[$ Myl06, Satz 14].

\section{The Discontinuity Problem in Pentagons}

In this section we investigate the discontinuity problem DIS by studying a number of stashingparallelization pentagons in which it appears as the bottom problem. Along the line we will formulate some problems that are equivalent to DIS. In the following we use the notation $f=\Sigma f$ for the stashing of specific problems $f$. We start with defining a number of problems related to $A C C_{\mathbb{N}}$.

Definition 5.1 (Problems related to all-or-co-unique choice). We consider the following problems:

(1) $A: \mathbb{N}^{\mathbb{N}} \rightrightarrows \mathbb{N},\langle\langle i, n\rangle, p\rangle \mapsto\left\{k \in \mathbb{N}: \varphi_{i}^{p}(n) \neq k\right\}$,

(2) $B: \mathbb{N}^{\mathbb{N}} \rightrightarrows \overline{\mathbb{N}}^{\mathbb{N}},\langle i, p\rangle \mapsto\left\{q \in \overline{\mathbb{N}}^{\mathbb{N}}:(\exists n) \varphi_{i}^{p}(n) \neq q(n) \in \mathbb{N}\right\}$,

(3) $C: \mathbb{N}^{\mathbb{N}} \rightrightarrows \mathbb{N}^{\mathbb{N}},\langle i, p\rangle \mapsto\left\{q \in \mathbb{N}^{\mathbb{N}}:(\exists n) \varphi_{i}^{p}(n) \neq q(n)\right\}$.

As a first result we prove that the discontinuity problem is the stashing of $\mathrm{ACC}_{\mathbb{N}}$.

Proposition 5.2 (All-or-co-unique choice). We obtain $\underline{\mathrm{ACC}_{\mathbb{N}}} \equiv_{\mathrm{sW}} \underset{\sim}{A} \equiv_{\mathrm{sW}} B \equiv_{\mathrm{sW}} C \equiv_{\mathrm{sW}}$ DIS and $\mathrm{ACC}_{\mathbb{N}} \equiv_{\mathrm{sW}} A$.

Proof. It is straightforward to see that $\mathrm{ACC}_{\mathbb{N}} \equiv_{\mathrm{sW}} A$, which implies $\mathrm{ACC}_{\mathbb{N}} \equiv_{\mathrm{sW}} \underset{\sim}{A}$ by Proposition 2.2 .

We prove $\underset{\sim}{A} \equiv_{\mathrm{sW}} B$. We note that $\underset{\sim}{A}$ is of type $\underset{\sim}{A}:\left(\mathbb{N}^{\mathbb{N}}\right)^{\mathbb{N}} \rightrightarrows \overline{\mathbb{N}}^{\mathbb{N}}$. In order to show $A \leq_{\mathrm{sW}} B$, we consider instances

$$
p=\left(\left\langle\left\langle i_{0}, n_{0}\right\rangle, p_{0}\right\rangle,\left\langle\left\langle i_{1}, n_{1}\right\rangle, p_{1}\right\rangle,\left\langle\left\langle i_{2}, n_{2}\right\rangle, p_{2}\right\rangle, \ldots\right) \in\left(\mathbb{N}^{\mathbb{N}}\right)^{\mathbb{N}}
$$

of $\underset{A}{A}$. There is a $j \in \mathbb{N}$ such that $\varphi_{j}^{\langle p\rangle}(k)=\varphi_{i_{k}}^{p_{k}}\left(n_{k}\right)$ for all $k \in \mathbb{N}$ and all $p$ of the above form. Hence, a solution to $B\langle j,\langle p\rangle\rangle$ is a solution to $A(p)$. Thus $A \leq_{\mathrm{sW}} B$. For the inverse reduction we consider the computable function $K: \mathbb{N}^{\mathbb{N}} \rightarrow\left(\mathbb{N}^{\mathbb{N}}\right)^{\widetilde{N}}$ with $K\langle i, p\rangle:=$ $(\langle\langle i, 0\rangle, p\rangle,\langle\langle i, 1\rangle, p\rangle,\langle\langle i, 2\rangle, p\rangle, \ldots)$. This function reduces $B$ to $\underset{\sim}{A}$, i.e., we obtain $\underset{\sim}{A} \equiv_{\mathrm{sW}} B$. 
It is easy to see that $B \leq_{\mathrm{sW}} C$ holds, since the parallelization $\widehat{\iota}: \mathbb{N}^{\mathbb{N}} \rightarrow \overline{\mathbb{N}}^{\mathbb{N}}$ of the embed$\operatorname{ding} \iota: \mathbb{N} \rightarrow \overline{\mathbb{N}}$ is computable, where $\iota$ is computable according to [BG20, Corollary 3.10].

We now prove $C \equiv_{\mathrm{sW}}$ DIS. The reduction $C \leq_{\mathrm{sW}}$ DIS follows with help of the computable function $K: \mathbb{N}^{\mathbb{N}} \rightarrow \mathbb{N}^{\mathbb{N}}$ with $K\langle i,\langle r, p\rangle\rangle:=\langle\langle i, r\rangle, p\rangle$. The computable inverse of $K$ yields DIS $\leq_{\mathrm{sW}} C$. We note that these reductions are obvious when the corresponding $\varphi_{i}^{\langle r, p\rangle}$ is total, but otherwise any $q \in \mathbb{N}^{\mathbb{N}}$ is allowed as a solution in both cases.

Finally, we prove DIS $\leq_{\mathrm{sW}} B$. By Theorem 1.9 it suffices to show that player I has a computable winning strategy in the Wadge game $B$. Therefore we consider a Gödel number $i \in \mathbb{N}$ such that $\varphi_{i}^{p}(n)=k$ if and only if $\langle n, k\rangle+1$ is the first number of the form $\langle n, m\rangle+1$ listed in $p$. If there is no number of this form, then $\varphi_{i}^{p}(n)$ is undefined. In other words, the program $i$ upon input $n$ and oracle $p$ searches for the first number of the form $\langle n, k\rangle+1$ listed in $p$ and outputs $k$ if such a number is found. Now player I in the Wadge game $B$ starts playing $\left\langle i, p_{0}\right\rangle$ with $p_{0}=000 \ldots$. This corresponds to the nowhere defined function $\varphi_{i}^{p_{0}}$ and hence player II must play a name of some $q_{0} \in \overline{\mathbb{N}}^{\mathbb{N}}$ with some $n \in \mathbb{N}$ such that $q_{0}(n) \in \mathbb{N}$; otherwise player II looses. However, when the first candidate $n_{0} \in \mathbb{N}$ with $q_{0}\left(n_{0}\right) \in \mathbb{N}$ appears, then player I modifies the $p_{0}$ in its play to a $p_{1}$ by appending $\left\langle n_{0}, q_{0}\left(n_{0}\right)\right\rangle$ in the current position to it. Hence $\varphi_{i}^{p_{1}}\left(n_{0}\right)=q_{0}\left(n_{0}\right)$. This forces player II to modify its play to a name of some $q_{1}$ with another $n_{1} \in \mathbb{N}$ with $q_{1}\left(n_{1}\right) \in \mathbb{N}$ and $n_{0} \neq n_{1}$; otherwise player II looses. Now player I modifies $p_{1}$ by appending $\left\langle n_{1}, q_{1}\left(n_{1}\right)\right\rangle$ to it. This strategy can be continued inductively and describes a computable winning strategy for player I.

We can conclude from Proposition 5.2 that the discontinuity problem is stashable.

Corollary 5.3. DIS is strongly stashable.

The next result supports the slogan that "non-computability is the parallelization of (effective) discontinuity".

Theorem 5.4 (Discontinuity and non-computability). $\widehat{\mathrm{DIS}} \equiv_{\mathrm{sW}}$ NON.

Proof. By Proposition 5.2 it suffices to show $\widehat{C} \equiv_{\mathrm{sW}}$ NON. For this purpose it is helpful to reformulate NON as follows:

$$
\mathrm{NON}: \mathbb{N}^{\mathbb{N}} \rightrightarrows \mathbb{N}^{\mathbb{N}}, p \mapsto\left\{q \in \mathbb{N}^{\mathbb{N}}:(\forall i)(\exists n) \varphi_{i}^{p}(n) \neq q(n)\right\}
$$

We note that this formulation of NON is equivalent to the usual one, as for non-total functions $\varphi_{i}^{p}$ there exists always an $n \in \mathbb{N} \backslash \operatorname{dom}\left(\varphi_{i}^{p}\right)$ that satisfies $\varphi_{i}^{p}(n) \neq q(n)$, since $q$ is total. With NON written in this form it is clear that $C \leq_{\mathrm{sW}}$ NON. Moreover, NON is strongly parallelizable, since given $p:=\left\langle p_{0}, p_{1}, \ldots\right\rangle$ it is clear that $q \mathbb{Z}_{\mathrm{T}} p$ implies $q \not_{\mathrm{T}} p_{i}$ for every $i \in \mathbb{N}$. Hence, $\widehat{C} \leq_{\mathrm{sW}} \mathrm{NON}$.

For the inverse reduction $\mathrm{NON} \leq{ }_{\mathrm{sW}} \widehat{C}$, we assume that we have given some $p \in \mathbb{N}^{\mathbb{N}}$. Then we can evaluate $\widehat{C}$ on the instance $\left(p_{i}\right)_{i \in \mathbb{N}}$ with $p_{i}:=\langle i, p\rangle$ in order to get some output $\left(q_{i}\right)_{i \in \mathbb{N}} \in \widehat{C}\left(p_{i}\right)_{i \in \mathbb{N}}$ with the property that $(\forall i)(\exists n) \varphi_{i}^{p}(n) \neq q_{i}(n)$. We claim that $q:=\left\langle q_{0}, q_{1}, q_{2}, \ldots\right\rangle \mathbb{X}_{\mathrm{T}} p$. If we assume the contrary, then there is some total computable $r: \mathbb{N} \rightarrow \mathbb{N}$ such that $\varphi_{r(i)}^{p}(n)=q_{i}(n)$ for all $i, n \in \mathbb{N}$. Hence, by the relativized version of Kleene's fixed point theorem [Soa16, Theorem 2.2.1] there is some $i \in \mathbb{N}$ with $\varphi_{i}^{p}(n)=$ $\varphi_{r(i)}^{p}(n)=q_{i}(n)$ for all $n \in \mathbb{N}$, which contradicts the assumption that $q_{i} \in C\langle i, p\rangle$. Altogether, this proves $\widehat{\mathrm{DIS}} \equiv_{\mathrm{sW}} \widehat{C} \equiv_{\mathrm{sW}} \mathrm{NON}$. 


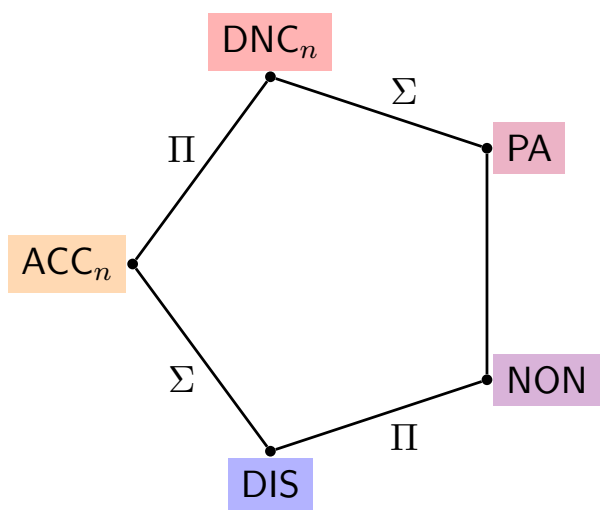

Figure 3: $\mathrm{ACC}_{n}$ pentagon in the Weihrauch lattice for $n \geq 2$.

We note that $\mathrm{DNC}_{\mathbb{N}}^{\mathcal{D}}<_{\mathrm{W}} \mathrm{DNC} C_{\mathbb{N}}$ follows since $A C C_{\mathbb{N}} \leq_{\mathrm{W}} \mathrm{DNC} C_{\mathbb{N}}$, but $A C C_{\mathbb{N}} \underline{Z}_{\mathrm{W}} \mathrm{DNC} C_{\mathbb{N}}^{\mathcal{D}}$ by Corollary 4.8. Together with Fact 1.10, we have established the pentagon of $A C C_{\mathbb{N}}$ given in Figure 1. Perhaps the pentagon in Figure 1 is the most natural pentagon in which the discontinuity problem DIS appears, but it is by far not the only one. It was observed by Jockusch [Joc89, Theorem 6] and Weihrauch [Wei92, Theorem 4.3] that the problems DNC $_{n}$ and $\mathrm{ACC}_{n}$, respectively, form strictly decreasing chains, i.e., we have the following fact (see also [HK14b, Corollary 82], [BHK17, Corollary 3.8]).

Fact 5.5 (Jockusch 1989, Weihrauch 1992). For all $n \geq 2$ we have:

(1) $\mathrm{DNC}_{\mathbb{N}}<_{\mathrm{sW}} \mathrm{DNC}_{n+1}<_{\mathrm{sW}} \mathrm{DNC}_{n}$,

(2) $\mathrm{ACC}_{\mathbb{N}}<_{\mathrm{sW}} \mathrm{ACC}_{n+1}<_{\mathrm{sW}} \mathrm{ACC}_{n}$.

On the other hand, it turns out that the stashing of the problem $A_{C C}$ is strongly equivalent to DIS for all $n \geq 2$. In order to express this result, it is useful to consider a universal function of type $\bigcup_{n}: \subseteq \mathbb{N}^{\mathbb{N}} \rightarrow\{0, \ldots, n-1\}^{\mathbb{N}}$. Such a function can be defined by truncating $\mathrm{U}$ accordingly:

$$
\mathrm{U}_{n}\langle\langle i, r\rangle, p\rangle:=\min \left(n-1, \varphi_{i}^{\langle r, p\rangle}\right)=\min (n-1, \mathrm{U}\langle\langle i, r\rangle, p\rangle)
$$

whenever $\varphi_{i}^{\langle r, p\rangle}$ is total (where the minimum is understood pointwise). Using this definition we can also modify the problem DIS accordingly and in this way we obtain

$$
\mathrm{DIS}_{n}: \mathbb{N}^{\mathbb{N}} \rightrightarrows\{0, \ldots, n-1\}^{\mathbb{N}}, p \mapsto\left\{q \in\{0, \ldots, n-1\}^{\mathbb{N}}: \mathrm{U}_{n}(p) \neq q\right\}
$$

for all $n \geq 2$. In these terms we obtain the following result.

Proposition 5.6 (All-or-co-unique choice). $\mathrm{ACC}_{n} \equiv_{\mathrm{sW}} \mathrm{DIS}_{n} \equiv_{\mathrm{sW}}$ DIS for all $n \geq 2$.

Proof. By Fact 5.5 it suffices to consider the case $n=2$. The remaining cases follow by Proposition 5.2 since stashing is an interior operator by Proposition 2.2. The reduction $\mathrm{DIS} \equiv_{\mathrm{sW}} \underline{\mathrm{ACC}_{\mathbb{N}}} \leq_{\mathrm{sW}} \mathrm{ACC}_{2}$ also follows. The reduction $\underline{\mathrm{ACC}_{2}} \leq_{\mathrm{sW}} \mathrm{DIS}_{2}$ can be proved almost literally following the lines of the proof of Proposition $\overline{5.2}$ with some obvious modifications. For instance, one needs to replace all terms $\varphi_{i}^{p}(n)$ in the definitions of $A, B$ and $C$ by $\min \left(1, \varphi_{i}^{p}(n)\right)$; one has to replace the output types of $A, B$ and $C$ by $\{0,1\}, \overline{\{0,1\}}^{\mathbb{N}}$ and $\{0,1\}^{\mathbb{N}}$, respectively; and one has to work with the embedding $\iota:\{0,1\} \rightarrow \overline{\{0,1\}}$. It remains to prove the reduction $\mathrm{DIS}_{2} \leq_{\mathrm{sW}}$ DIS. For this direction we use the computable embedding 


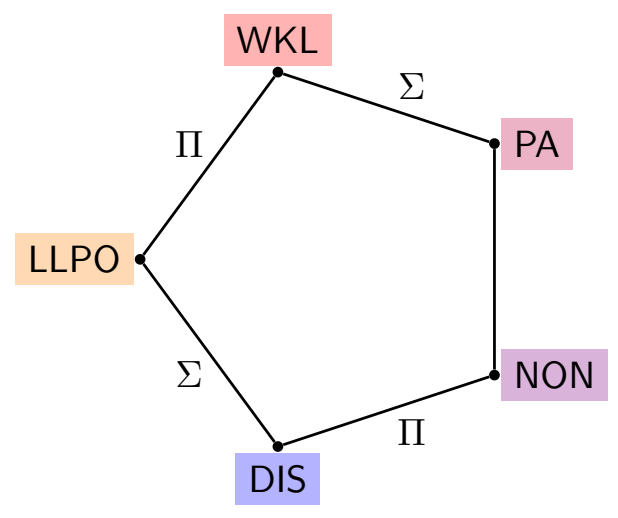

Figure 4: LLPO pentagon in the Weihrauch lattice.

$\iota: \mathbb{N}^{\mathbb{N}} \rightarrow 2^{\mathbb{N}}, p \mapsto 0^{p(0)} 10^{p(1)} 10^{p(2)} 1 \ldots$. There is a total computable $s: \mathbb{N} \rightarrow \mathbb{N}$ such that $\iota\left(\varphi_{s(i)}^{t}\right)=\min \left(1, \varphi_{i}^{t}\right)$ for all $i \in \mathbb{N}$ and $t \in \mathbb{N}^{\mathbb{N}}$ such that $\varphi_{i}^{t}$ is total and $\min \left(1, \varphi_{i}^{t}\right)$ contains infinitely many ones. Now, given an instance $\langle\langle i, r\rangle, p\rangle$ of $\mathrm{DIS}_{2}$ we compute the instance $\langle\langle s(i), r\rangle, p\rangle$ of DIS. If $q \in \mathbb{N}^{\mathbb{N}}$ satisfies $q \neq \mathrm{U}\langle\langle s(i), r\rangle, p\rangle$, then $\iota(q) \neq \mathrm{U}_{2}\langle\langle i, r\rangle, p\rangle$ follows. This is clear if $\varphi_{i}^{\langle r, p\rangle}$ is total and $\min \left(1, \varphi_{i}^{\langle r, p\rangle}\right)$ contains infinitely many ones. But otherwise every $q \in \mathbb{N}^{\mathbb{N}}$ satisfies the conclusion. Altogether, this completes the proof.

The upper Turing cone version of $\mathrm{DNC}_{n}$ for $n \geq 2$ is just the problem PA of finding a PA degree relative to the input. By a result of Jockusch and Friedberg [Joc89, Theorem 5] the Turing degrees of $q \gg p$ are exactly the degrees that compute a diagonally non-computable function $f: \mathbb{N} \rightarrow\{0, \ldots, n-1\}$ relative to $p$ for every $n \geq 2$ (see also [BHK17, Proposition 6.1]). Hence we obtain [BHK17, Corollary 6.4] the following fact.

Fact 5.7. $\mathrm{PA} \equiv_{\mathrm{sW}} \mathrm{DNC}_{n}^{\mathcal{D}}$ for every $n \geq 2$.

We note that PA $<_{\mathrm{W}} \mathrm{DNC}_{n}$ follows since $\mathrm{ACC}_{n} \leq_{\mathrm{W}} \mathrm{DNC}_{n}$, but $\mathrm{ACC}_{n} \mathbb{L}_{\mathrm{W}}$ PA by Corollary 4.7. Altogether, we have thus established the pentagon of $\mathrm{ACC}_{n}$ for $n \geq 2$ given in Figure 3. An important special case of this diagram is the case for $n=2$. Since $\mathrm{LLPO} \equiv{ }_{\mathrm{sW}} \mathrm{ACC}_{2}$, Fact 1.10 yields the stashing-parallelization pentagon of LLPO given in Figure 4 .

We have a number of basic discrete problems ordered in the following way [BHK17, Fact 3.4], [BG11a, Theorem 3.10], [BGM12, Section 13].

Fact 5.8. $A C C_{\mathbb{N}} \leq_{W} L L P O \leq_{W} L P O \leq_{W} \lim _{2} \leq_{W} C_{\mathbb{N}}$.

Now the question appears how far up in this chain of discrete problems we can go such that we still obtain the discontinuity problem DIS as stashing of the corresponding discrete problem? We will see in Proposition 8.3 that a phase transition in this respect happens between LPO and $\lim _{2}$.

We now study the pentagon of LPO. We use the notation $W_{i}^{p}:=\operatorname{dom}\left(\varphi_{i}^{p}\right)$ and by $\chi_{A}: \mathbb{N} \rightarrow\{0,1\}$ we denote the characteristic function of $A \subseteq \mathbb{N}$ with $A=\chi_{A}^{-1}\{1\}$. We first define some problems related to LPO.

Definition 5.9 (Problems related to LPO). We consider:

(1) $L: \mathbb{N}^{\mathbb{N}} \rightarrow\{0,1\},\langle\langle i, n\rangle, p\rangle \mapsto 1-\chi_{\mathrm{W}_{i}^{p}}(n)=\left\{\begin{array}{ll}0 & \text { if } n \in \operatorname{dom}\left(\varphi_{i}^{p}\right) \\ 1 & \text { otherwise }\end{array}\right.$ ， 
(2) $D: \mathbb{N}^{\mathbb{N}} \rightrightarrows \overline{\{0,1\}}^{\mathbb{N}},\langle i, p\rangle \mapsto\left\{q \in \overline{\{0,1\}}^{\mathbb{N}}:(\exists n) \chi_{\mathrm{W}_{i}^{p}}(n) \neq q(n) \in\{0,1\}\right\}$,

(3) $E: \mathbb{N}^{\mathbb{N}} \rightrightarrows\{0,1\}^{\mathbb{N}},\langle i, p\rangle \mapsto\left\{q \in\{0,1\}^{\mathbb{N}}:(\exists n) \chi_{\mathrm{W}_{i}^{p}}(n) \neq q(n)\right\}$

Now we can prove the following result.

Proposition 5.10 (Stashing of LPO). LPO $\equiv_{\mathrm{sW}} L$ and $\underline{\mathrm{LPO}} \equiv_{\mathrm{sW}} \underset{\sim}{L} \equiv_{\mathrm{sW}} D \equiv_{\mathrm{sW}} E \equiv_{\mathrm{sW}}$ DIS.

Proof. We proceed as in the proof of Proposition 5.2. It is easy to see that $\mathrm{LPO} \equiv_{\mathrm{sW}} L$, which implies $\underline{\mathrm{LPO}} \equiv_{\mathrm{sW}} L$. The same reductions that prove $A \equiv_{\mathrm{sW}} B$ in Proposition 5.2 also show $L \equiv_{\mathrm{sW}} D$. The reduction $D \leq_{\mathrm{sW}} E$ follows using the computable embedding $\iota:\{0,1\} \rightarrow\{0,1\}$. By Proposition 5.2 and Fact 5.8 and since stashing is an interior operator by Proposition 2.2 , we obtain DIS $\equiv_{\mathrm{sW}} \mathrm{ACC}_{\mathbb{N}} \leq_{\mathrm{sW}} \mathrm{LPO}$.

It only remains to show $E \leq_{\mathrm{sW}}$ DIS. By the proof of Proposition 5.6 it suffices to show $E \leq_{\mathrm{sW}} C$, where

$$
C: \mathbb{N}^{\mathbb{N}} \rightrightarrows\{0,1\}^{\mathbb{N}},\langle i, p\rangle \mapsto\left\{q \in\{0,1\}^{\mathbb{N}}:(\exists n) \min \left(1, \varphi_{i}^{p}(n)\right) \neq q(n)\right\}
$$

is the modification of the function from Proposition 5.2 that was used in the proof of Proposition 5.6 in order to show DIS $\equiv_{\mathrm{sW}} \mathrm{DIS}_{2} \equiv_{\mathrm{sW}} C$. For the reduction $E \leq_{\mathrm{sW}} C$ we proceed as follows. Given an instance $\langle i, p\rangle$ of $E$, we try to find out for each $n \in \mathbb{N}$, which of the two consecutive values $2 n, 2 n+1$ appears in $\mathbf{W}_{i}^{p}$ first, if any. More precisely, there is a computable function $r: \mathbb{N} \rightarrow \mathbb{N}$ such that

$$
\varphi_{r(i)}^{p}(n)= \begin{cases}0 & \text { if } 2 n \in \mathbf{W}_{i}^{p} \text { is found first } \\ 1 & \text { if } 2 n+1 \in \mathbf{W}_{i}^{p} \text { is found first } \\ \uparrow & \text { if }\{2 n, 2 n+1\} \cap \mathbf{W}_{i}^{p}=\emptyset\end{cases}
$$

holds for all $i, n \in \mathbb{N}$ and $p \in \mathbb{N}^{\mathbb{N}}$. We use the computable function $K: \mathbb{N}^{\mathbb{N}} \rightarrow \mathbb{N}^{\mathbb{N}}$ with $K\langle i, p\rangle=\langle r(i), p\rangle$ to translate instances of $E$ into instances of $C$ and the computable function $H:\{0,1\}^{\mathbb{N}} \rightarrow\{0,1\}^{\mathbb{N}}$ with $H(q)(2 n):=1-q(n)$ and $H(q)(2 n+1):=q(n)$ in order to translate solutions of $C$ into solutions of $E$. That this reduction is correct can be seen as follows. Given an instance $\langle i, p\rangle$ of $E$ and $q \in C\langle r(i), p\rangle=C K\langle i, p\rangle$, there is some $n \in \mathbb{N}$ with $\min \left(1, \varphi_{r(i)}^{p}(n)\right) \neq q(n)$. We are now in exactly one of the following three cases:

(1) $\varphi_{r(i)}^{p}(n)=0 \Longrightarrow\left(q(n)=1\right.$ and $\left.2 n \in \mathrm{W}_{i}^{p}\right) \Longrightarrow H(q)(2 n)=0 \neq \chi_{\mathrm{W}_{i}^{p}}(2 n)$,

(2) $\varphi_{r(i)}^{p(n)}=1 \Longrightarrow\left(q(n)=0\right.$ and $\left.2 n+1 \in \mathrm{W}_{i}^{p}\right)$

$\Longrightarrow H(q)(2 n+1)=0 \neq \chi_{\mathrm{W}_{i}^{p}}(2 n+1)$,

(3) $\varphi_{r(i)}^{p}(n)=\uparrow \Longrightarrow\left(q(n) \in\{0,1\}\right.$ and $\left.\{2 n, 2 n+1\} \cap \mathrm{W}_{i}^{p}=\emptyset\right)$

$\Longrightarrow\left(H(q)(2 n)=1 \neq \chi_{\mathrm{W}_{i}^{p}}(2 n)\right.$ or $\left.H(q)(2 n+1)=1 \neq \chi_{\mathrm{W}_{i}^{p}}(2 n+1)\right)$.

In any case we obtain $H(q) \in E\langle i, p\rangle$. Altogether we obtain $E \leq_{\mathrm{sW}} C \equiv_{\mathrm{sW}}$ DIS.

By Fact 1.10 we have $\widehat{\mathrm{LPO}} \equiv_{\mathrm{sW}} \lim \equiv_{\mathrm{sW}} \mathrm{J}$. It is clear that $\mathrm{J}^{\mathcal{D}}$ is strongly Weihrauch equivalent to

$$
\mathrm{J}^{\mathcal{D}}: \mathcal{D} \rightrightarrows \mathcal{D}, a \mapsto\left\{b \in \mathcal{D}: a^{\prime} \leq_{\mathrm{T}} b\right\}
$$

We note that $\mathrm{J}^{\mathcal{D}}<_{\mathrm{W}} \lim$ follows since $\mathrm{LPO} \leq_{\mathrm{W}} \lim$, but LPO $\not_{\mathrm{W}} \mathrm{J}^{\mathcal{D}}$ by Corollary 4.7. Altogether, we have established the pentagon of LPO given in Figure 5.

Proposition 5.10 also leads to another characterization of the discontinuity problem in terms of ranges. This characterization is unique among all the characterizations that we have provided because it is purely set-theoretic (i.e., no Gödel numberings or other computability-theoretic concepts are used) and because it only involves standard data types (i.e., no completions are mentioned). 


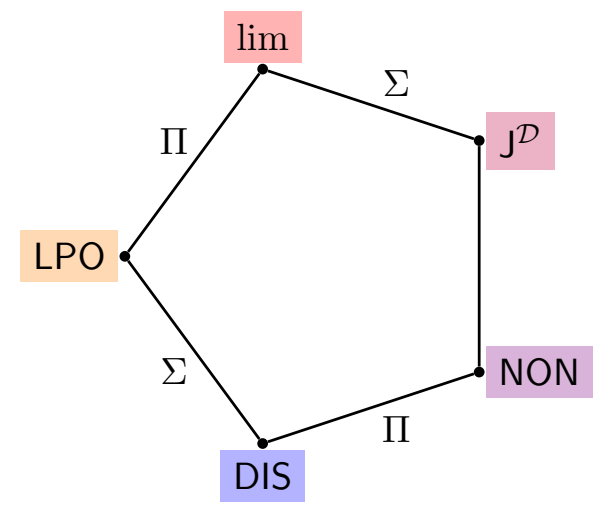

Figure 5: LPO pentagon in the Weihrauch lattice.

Definition 5.11 (Range non-equality problem). We call

$$
\text { NRNG : } \mathbb{N}^{\mathbb{N}} \rightrightarrows 2^{\mathbb{N}}, p \mapsto\left\{A \in 2^{\mathbb{N}}: A \neq \operatorname{range}(p-1)\right\}
$$

the range non-equality problem.

We now obtain the following characterization.

Corollary 5.12 (Range non-equality problem). DIS $\equiv_{\mathrm{sW}}$ NRNG.

Proof. By Proposition 5.10 it suffices to show NRNG $\equiv_{\mathrm{sW}} E$. For one, there is a $j \in \mathbb{N}$ such that $\mathrm{W}_{j}^{p}=\operatorname{range}(p-1)$, which establishes the reduction $N R N G \leq_{\mathrm{sW}} E$. On the other hand, there is a computable $f: \mathbb{N}^{\mathbb{N}} \rightarrow \mathbb{N}^{\mathbb{N}}$ such that range $(f\langle i, p\rangle-1)=W_{i}^{p}$ for all $p \in \mathbb{N}^{\mathbb{N}}$ and $i \in \mathbb{N}$, which shows $E \leq_{\mathrm{sW}}$ NRNG.

The reader might have noticed that a lot of problems that occur in the lower parts of our pentagons can actually be seen as complementary problems of other well-known problems. We briefly make this more precise.

Definition 5.13 (Complementary problem). For every problem $f: \subseteq X \rightrightarrows Y$ we define the complementary problem $f^{\mathrm{c}}: \subseteq X \rightrightarrows Y$ by $\operatorname{graph}\left(f^{\mathrm{c}}\right):=\operatorname{graph}(f)^{\mathrm{c}}=(X \times Y) \backslash \operatorname{graph}(f)$.

That is $\operatorname{dom}\left(f^{\mathrm{c}}\right)=\{x \in X: f(x) \neq Y\}$ and $f(x):=Y \backslash f(x)$ for all $x \in \operatorname{dom}\left(f^{\mathrm{c}}\right)$. Using this concept we see that DIS $=\mathrm{U}^{\mathrm{c}}, \mathrm{NRNG}=\mathrm{EC}^{\mathrm{c}}$, and NON $=\left(\geq_{\mathrm{T}}\right)^{\mathrm{c}}$, where $\geq_{\mathrm{T}}: \mathbb{N}^{\mathbb{N}} \rightrightarrows$ $\mathbb{N}^{\mathbb{N}}, p \mapsto\left\{q \in \mathbb{N}^{\mathbb{N}}: q \leq_{\mathrm{T}} p\right\}$.

Even though complementation yields a neat way of expressing these problems, $f \mapsto f^{\mathrm{c}}$ is not an operation on the Weihrauch lattice. For instance $\mathrm{J}^{\mathrm{c}}$ is obviously computable, whereas $\mathrm{EC}^{\mathrm{C}} \equiv_{\mathrm{sW}}$ DIS is not, although $\mathrm{J} \equiv_{\mathrm{sW}} \mathrm{EC}$ by Fact 1.10 .

\section{MAJORIZATION AND HYPERIMMUnity}

In this section we study the stashing-parallelization pentagons of the non-majorization problem NMAJ that can be seen as an asymmetric version of the discontinuity problem. The non-majorization problem NMAJ is introduced in the following definition and it is related to the well-known hyperimmunity problem.

Definition 6.1 (Problems related to hyperimmunity). We consider the following problems: (1) NGEQ: $\mathbb{N}^{\mathbb{N}} \rightrightarrows \mathbb{N},\langle\langle i, n\rangle, p\rangle \mapsto\left\{k \in \mathbb{N}: \varphi_{i}^{p}(n) \nsupseteq k\right\}$, 
(2) NMAJ : $\mathbb{N}^{\mathbb{N}} \rightrightarrows \mathbb{N}^{\mathbb{N}},\langle i, p\rangle \mapsto\left\{q \in \mathbb{N}^{\mathbb{N}}:(\exists n) \varphi_{i}^{p}(n) \nsucceq q(n)\right\}$,

(3) HYP : $\mathbb{N}^{\mathbb{N}} \rightrightarrows \mathbb{N}^{\mathbb{N}}, p \mapsto\left\{q \in \mathbb{N}^{\mathbb{N}}:\left(\forall r \leq_{\mathrm{T}} p\right)(\exists n) r(n)<q(n)\right\}$,

(4) MEET : $\mathbb{N}^{\mathbb{N}} \rightrightarrows \mathbb{N}^{\mathbb{N}}, p \mapsto\left\{q \in \mathbb{N}^{\mathbb{N}}:\left(\forall r \leq_{\mathrm{T}} p\right)(\exists n) r(n)=q(n)\right\}$,

(5) 1-WGEN : $\mathbb{N}^{\mathbb{N}} \rightrightarrows \mathbb{N}^{\mathbb{N}}, p \mapsto\left\{q \in \mathbb{N}^{\mathbb{N}}: q\right.$ is weakly 1 -generic relative to $\left.p\right\}$.

The non-majorization problem NMAJ has been defined here ad hoc, whereas the Weihrauch complexity of the hyperimmunity problem HYP and the weak 1-genericity problem 1-WGEN have already been studied in [BHK17, BHK18]. The principle MEET was introduced in a reverse mathematics context in [HRSZ17]. We note that the existential quantifier " $\exists n$ " in HYP and MEET could equivalently be replaced by " $\exists^{\infty} n$ ". We recall that a point $p \in \mathbb{N}^{\mathbb{N}}$ is called weakly 1 -generic relative to $q \in \mathbb{N}^{\mathbb{N}}$ if $p \in U$ for every dense open set $U \subseteq \mathbb{N}^{\mathbb{N}}$ that is c.e. open relative to $q$. A set $U \subseteq \mathbb{N}^{\mathbb{N}}$ is c.e. open relative to $q$ if $U=U_{i}^{q}:=\left\{p \in \mathbb{N}^{\mathbb{N}}: 0 \in \operatorname{dom}\left(\varphi_{i}^{q}\right)\right\}$ for some $i \in \mathbb{N}$. By a theorem of Kurtz the hyperimmune degrees coincide with the weakly 1-generic degrees and this also holds uniformly in the following sense [BHK17, Corollary 9.5].

Fact 6.2 (Uniform theorem of Kurtz). HYP $\equiv_{W} 1$-WGEN.

On the first sight, the non-majorization problem NMAJ looks similar to the discontinuity problem DIS in the form of $C$, as defined in Definition 5.1. In fact, NMAJ can be seen as an asymmetric version of $C$, since the inequality $\neq$ is simply replaced by $¥$ (we note that $¥$ is not the same as $<$ here, as $\varphi_{i}^{p}$ might be partial and $\varphi_{i}^{p}(n) \nsupseteq q(n)$ is supposed to mean that either $\varphi_{i}^{p}(n)$ does not exist or $\varphi_{i}^{p}(n)$ exists and $\varphi_{i}^{p}(n)<q(n)$.) Despite the similarity between NMAJ and DIS, it turns out that NMAJ is neither equivalent to DIS nor stashable. Among all the problems that we have studied here, it is perhaps the one that comes closest to DIS without being equivalent to it. The following result clarifies the relation of these problems to each other.

Proposition 6.3 (The non-majorization problem). We obtain $\underline{N M A J} \equiv_{\mathrm{W}} \underline{\mathrm{NGEQ}} \equiv_{\mathrm{W}}$ DIS, $\widehat{\mathrm{NMAJ}} \equiv{ }_{\mathrm{SW}} \mathrm{HYP}, \mathrm{DIS}<_{\mathrm{W}} \mathrm{NMAJ}<_{\mathrm{W}} \mathrm{HYP}$, and $\mathrm{NON}<_{\mathrm{W}} \mathrm{HYP}^{\mathcal{D}}$. In particular, NMAJ is not stashable.

Proof. With $C$ from Definition 5.1 we obtain DIS $\equiv_{\mathrm{sW}} C \leq_{\mathrm{sW}} \mathrm{NMAJ} \leq_{\mathrm{sW}} \mathrm{NGEQ} \leq_{\mathrm{W}} \mathrm{LPO}$. The latter reduction holds since LPO can be used to determine whether $\varphi_{i}^{p}(n)$ is defined and if it is defined then one can use the original input to find a larger value; otherwise 0 is a suitable output. This implies DIS $\equiv_{\mathrm{W}} \mathrm{NGEQ} \equiv_{\mathrm{W}}$ NMAJ by Proposition 5.10 since stashing is an interior operator by Proposition 2.2. Moreover, we have

$$
\operatorname{HYP}(p)=\left\{q \in \mathbb{N}^{\mathbb{N}}:\left(\forall r \leq_{\mathrm{T}} p\right)(\exists n) r(n)<q(n)\right\}=\left\{q \in \mathbb{N}^{\mathbb{N}}:(\forall i)(\exists n) \varphi_{i}^{p}(n) \nsupseteq q(n)\right\} .
$$

Here clearly " $\supseteq$ " holds regarding the second equality since the $\varphi_{i}^{p}$ include all the total $r \leq_{\mathrm{T}} p$ and the other inclusion " $\subseteq$ " holds as for $\varphi_{i}^{p}$ that are not total the condition $\varphi_{i}^{p}(n) \nsupseteq q(n)$ is satisfied by definition for all $n \notin \operatorname{dom}\left(\varphi_{i}^{p}\right)$. We claim that $\widehat{\mathrm{NMAJ}} \equiv_{\mathrm{sW}} \mathrm{HYP}$. For one, it is clear that NMAJ $\leq_{\mathrm{sW}} \mathrm{HYP}$ holds. Moreover, HYP is strongly parallelizable, as there is some computable $r: \mathbb{N} \rightarrow \mathbb{N}$ with $\varphi_{r\langle i, k\rangle}^{\left\langle p_{0}, p_{1}, p_{2}, \ldots\right\rangle}(n)=\varphi_{i}^{p_{k}}(n)$ for all $i, n, k \in \mathbb{N}$ and $p_{0}, p_{1}, \ldots \in \mathbb{N}^{\mathbb{N}}$. Together, this implies $\widehat{\mathrm{NMAJ}} \leq_{\mathrm{sW}} \mathrm{HYP}$. On the other hand, there is a computable $s: \mathbb{N} \rightarrow \mathbb{N}$ such that $\varphi_{s(i)}^{p}(n)=\varphi_{i}^{p}\langle i, n\rangle$ for all $i, n \in \mathbb{N}$ and $p \in \mathbb{N}^{\mathbb{N}}$. Hence, the function $K: \mathbb{N}^{\mathbb{N}} \rightarrow \mathbb{N}^{\mathbb{N}}$ with $K(p):=\langle\langle s(0), p\rangle,\langle s(1), p\rangle,\langle s(2), p\rangle, \ldots\rangle$ is computable and with 


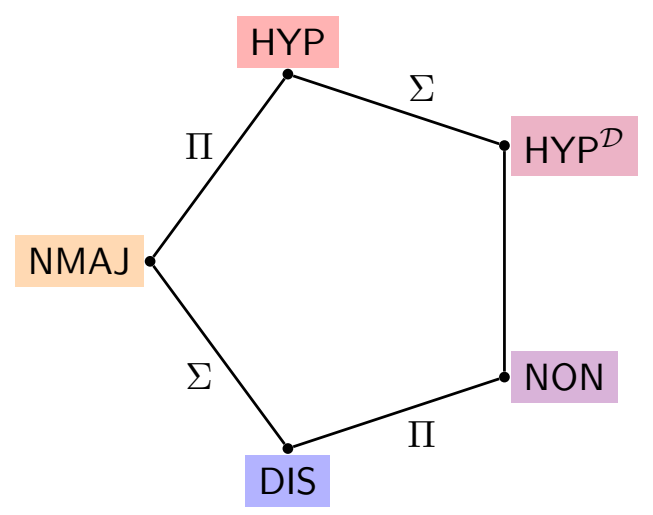

Figure 6: Non-marjorization pentagon in the Weihrauch lattice.

$$
\begin{aligned}
& q=\left\langle q_{0}, q_{1}, q_{2}, \ldots\right\rangle \in \widehat{\mathrm{NMAJ}} \circ K(p) \text { we obtain } \\
& \qquad(\forall i)(\exists n) \varphi_{i}^{p}\langle i, n\rangle=\varphi_{s(i)}^{p}(n) \nsupseteq q_{i}(n)=q\langle i, n\rangle,
\end{aligned}
$$

so in particular $q \in \operatorname{HYP}(p)$. This proves $\mathrm{HYP} \leq_{\mathrm{sW}} \widehat{\mathrm{NMAJ}}$.

Suppose DIS $\equiv_{\mathrm{W}}$ NMAJ, then NON $\equiv_{\mathrm{W}}$ HYP would follow by Theorem 5.4 , since parallelization is a closure operator. However, it is well-known that there are hyperimmune-free non-computable degrees [MM68, Section 2], i.e., there is non-computable $q$ which is not of hyperimmune degree. The problem NON has a realizer that, on computable inputs, produces such non-computable $q$, which is not of hyperimmune degree. Since hyperimmune degrees are upwards closed by [MM68, Theorem 1.1], we obtain that NON $<_{W} \mathrm{HYP}^{\mathcal{D}} \leq_{W} \mathrm{HYP}$. This implies DIS $<_{W}$ NMAJ. Finally, we clearly have NMAJ $<_{W} \mathrm{HYP}$ as NMAJ has computable solutions on all instances, while HYP does not.

We emphasize that we have only proved NMAJ $\equiv_{\mathrm{W}}$ DIS with an ordinary Weihrauch equivalence, unlike in all previous cases, where we have established a strong Weihrauch equivalence. Hence we are left with the following open question.

Question 6.4. Does NMAJ $\equiv_{\mathrm{sW}}$ DIS hold?

We note that $\mathrm{HYP}^{\mathcal{D}}$ is exactly the problem of finding a hyperimmune degree relative to the input, i.e., it can equivalently be described as

$$
\operatorname{HYP}^{\mathcal{D}}: \mathcal{D} \rightrightarrows \mathcal{D}, a \mapsto\{b \in \mathcal{D}: b \text { is of hyperimmune degree relative to } a\}
$$

since hyperimmune degrees are upwards closed by [MM68, Theorem 1.1]. This establishes the pentagon in Figure 6, except that we did not yet prove $\mathrm{HYP}^{\mathcal{D}}<_{\mathrm{W}} \mathrm{HYP}$. In the case of the earlier pentagons discussed here, we have used Corollary 4.7 for the corresponding separation. In the case of the hyperimmunity problem a more tailor-made argument is required, since NMAJ has no natural number output and is densely realized itself. We combine ideas from the proof of [BP18, Proposition 6.3] and the proof of Proposition 5.2.

Proposition 6.5. $\mathrm{HYP}^{\mathcal{D}}<_{\mathrm{W}} \mathrm{HYP}$ (even restricted to computable instances).

Proof. Here we consider $\mathrm{HYP}^{\mathcal{D}}$ to be defined as

$$
\operatorname{HYP}^{\mathcal{D}}: \mathbb{N}^{\mathbb{N}} \rightrightarrows \mathbb{N}^{\mathbb{N}}, p \mapsto\left\{s \in \mathbb{N}^{\mathbb{N}}:\left(\exists q \leq_{\mathrm{T}} s\right)\left(\forall r \leq_{\mathrm{T}} p\right)(\exists n) r(n)<q(n)\right\} .
$$


It suffices to prove NMAJ $\not \mathrm{W}_{\mathrm{W}} \mathrm{HYP}^{\mathcal{D}}$. Let us assume the contrary, i.e., let $H, K: \subseteq \mathbb{N}^{\mathbb{N}} \rightarrow \mathbb{N}^{\mathbb{N}}$ be computable functions such that $H\langle\mathrm{id}, G K\rangle$ is a realizer of NMAJ whenever $G$ is a realizer of HYP ${ }^{\mathcal{D}}$. Then there is a computable monotone function $h: \mathbb{N}^{*} \rightarrow \mathbb{N}^{*}$ that approximates $H$ in the sense that $H(q)=\sup _{w \sqsubseteq q} h(w)$ for all $q \in \operatorname{dom}(H)$. As in the proof of Proposition 5.2 we consider a fixed Gödel number $i \in \mathbb{N}$ such that $\varphi_{i}^{p}(n)=k$ if and only if $\langle n, k\rangle+1$ is the first number of the form $\langle n, m\rangle+1$ listed in $p$. Now we use $h$ and the finite extension method to construct an input $i p=\langle i, p\rangle \in \mathbb{N}^{\mathbb{N}}$ of NMAJ on which the above reduction fails. We start with $p_{0}:=000 \ldots$, which yields the nowhere defined function $\varphi_{i}^{p_{0}}$. For this $p_{0}$ there is a lexicographically first $w_{0} \in\{2,3,4, \ldots\}^{*}$ such that $\left|h\left\langle\left. i p_{0}\right|_{\left|w_{0}\right|}, d_{0} w_{0}\right\rangle\right|>0$ for both values $d_{0} \in\{0,1\}$. This is because there is some $s \in\{2,3,4, \ldots\}^{\mathbb{N}}$ with $d_{0} s \in \operatorname{HYP}^{\mathcal{D}} K\left\langle i, p_{0}\right\rangle$ for both values $d_{0} \in\{0,1\}$. We choose

$$
a_{0}:=\max \left\{h\left\langle\left. i p_{0}\right|_{\left|w_{0}\right|}, d_{0} w_{0}\right\rangle(0): d_{0} \in\{0,1\}\right\} \text { and } b_{0}:=\left\langle 0, a_{0}\right\rangle+1
$$

and we continue with $p_{1}:=0^{\left|w_{0}\right|} b_{0} 000 \ldots$, which yields a function that satisfies $\varphi_{i}^{p_{1}}(0)=a_{0}$ and is undefined otherwise. Again there is a lexicographically first $w_{1} \in\{2,3,4, \ldots\}^{*}$ such that $\left|h\left\langle\left. i p_{1}\right|_{\left|w_{0}\right|+\left|w_{1}\right|+1}, d_{0} w_{0} d_{1} w_{1}\right\rangle\right|>1$ for all values $d_{0}, d_{1} \in\{0,1\}$ and now we choose

$$
a_{1}:=\max \left\{h\left\langle\left. i p_{1}\right|_{\left|w_{0}\right|+\left|w_{1}\right|+1}, d_{0} w_{0} d_{1} w_{1}\right\rangle(1): d_{0}, d_{1} \in\{0,1\}\right\} \text { and } b_{1}:=\left\langle 1, a_{1}\right\rangle+1 .
$$

The next input is $p_{2}:=0^{\left|w_{0}\right|} b_{0} 0^{\left|w_{1}\right|} b_{1} 000 \ldots$, which represents a function $\varphi_{i}^{p_{2}}$ that satisfies $\varphi_{i}^{p_{2}}(j)=a_{j}$ for $j \in\{0,1\}$ and that is undefined otherwise. We continue the construction inductively and obtain computable sequences $\left(p_{n}\right)_{n \in \mathbb{N}}$ in $\mathbb{N}^{\mathbb{N}}$ and $\left(w_{n}\right)_{n \in \mathbb{N}}$ in $\mathbb{N}^{*}$ in this way. The sequence $\left(p_{n}\right)_{n \in \mathbb{N}}$ converges to a computable $p \in \mathbb{N}^{\mathbb{N}}$. This is because the construction above only depends on the computable function $h$ and yields longer and longer portions of $p$. For every $d \in\{0,1\}^{\mathbb{N}}$ we denote by $s_{d}$ the sequence $s_{d}:=d_{0} w_{0} d_{1} w_{1} d_{2} w_{2} \ldots$ with $d_{j}:=d(j)$. The construction ensures that $H\left\langle i p, s_{d}\right\rangle(n) \leq \varphi_{i}^{p}(n)$ for every $n \in \mathbb{N}$ and $d \in\{0,1\}^{\mathbb{N}}$ and hence $H\left\langle\langle i, p\rangle, s_{d}\right\rangle \notin \operatorname{NMAJ}\langle i, p\rangle$ for every $d \in\{0,1\}^{\mathbb{N}}$. On the other hand, there is some $d \in\{0,1\}^{\mathbb{N}}$ of hyperimmune degree, which implies $s_{d} \in \mathrm{HYP}^{\mathcal{D}} K\langle i, p\rangle$, since $d \leq_{\mathrm{T}} s_{d}$. This yields a contradiction to the assumption and hence $\mathrm{HYP}^{\mathcal{D}}<\mathrm{W} H Y P$.

Hence, there is no uniform computable method to find a hyperimmune $q \in \mathbb{N}^{\mathbb{N}}$ from an arbitrary member of a hyperimmune degree. This also yields a second proof of NMAJ $\mathbb{K}_{\mathrm{W}}$ DIS. Finally, we mention that the separation in Proposition 6.5 also yields a separation of the corresponding problems (i.e., the sets given by the respective solutions on computable instances) in the Medvedev lattice (see [BGP21, Theorem 9.1]).

We now want to show that MEET is equivalent to HYP. The corresponding proof of [HRSZ17, Theorem 38] can be transferred into our setting. On the first sight it might be a bit surprising that replacing $\neq$ by $¥$ makes a difference, while replacing $<$ by $=$ does not. However, this comparison does not take the aspect of totality into account. For completeness we include the proof, which is interesting by itself.

Proposition 6.6. HYP $\equiv_{\mathrm{W}}$ MEET.

Proof. For $p \in \mathbb{N}^{\mathbb{N}}$ we have

$$
\operatorname{MEET}(p)=\left\{q \in \mathbb{N}^{\mathbb{N}}:(\forall i)\left(\varphi_{i}^{p} \text { total } \Longrightarrow(\exists n) \varphi_{i}^{p}(n)=q(n)\right)\right\}
$$

We note that in the case of HYP we obtain a corresponding formulation with $\nsucceq$ instead of $=$. In this case totality does not need to be mentioned as the negative condition is automatically satisfied by partial $\varphi_{i}^{p}$. It is obvious that HYP $\leq_{\mathrm{sW}}$ MEET, as we just have to use $q \mapsto q+1$ 
to translate the solution on the same input $p$. For the opposite direction we note that by the smn-theorem there is a computable $s: \mathbb{N} \rightarrow \mathbb{N}$ such that $\varphi_{s(i)}^{p}(n)=\Phi_{i}^{p}\langle i, n\rangle$ for all $i, n \in \mathbb{N}$. Here $\Phi_{i}^{p}(n)$ denotes the time complexity, i.e., the number of computation steps required to compute $\varphi_{i}^{p}(n)$ (if it exists and undefined otherwise). Then given an input $p \in \mathbb{N}^{\mathbb{N}}$ and $q \in \operatorname{HYP}(p)$ we can compute $r \in \mathbb{N}^{\mathbb{N}}$ with

$$
r\langle i, n\rangle:= \begin{cases}\varphi_{i}^{p}\langle i, n\rangle & \text { if } \Phi_{i}^{p}\langle i, n\rangle<q(n) \\ 0 & \text { otherwise }\end{cases}
$$

for all $i, n \in \mathbb{N}$. If $i \in \mathbb{N}$ is such that $\varphi_{i}^{p}$ is total, then $\Phi_{i}^{p}$ and hence $\varphi_{s(i)}^{p}$ are total too and hence there is some $n \in \mathbb{N}$ with $\Phi_{i}^{p}\langle i, n\rangle=\varphi_{s(i)}^{p}(n)<q(n)$. This implies that $r\langle i, n\rangle=\varphi_{i}^{p}\langle i, n\rangle$, i.e., $r \in \operatorname{MEET}(p)$. Since $r$ can be computed, given $p, q$, we obtain MEET $\leq_{\mathrm{W}} \mathrm{HYP}$.

We note that the backwards reduction is not a strong Weihrauch reduction.

\section{Retractions, All-or-Unique Choice and Gödel Numbers}

In the previous sections we have discussed the stashing of a number of problems of type $f: \subseteq X \rightrightarrows \mathbb{N}$ with natural number output. In this particular situation we can also describe stashing in an alternative way. This is because the space $\overline{\mathbb{N}}^{\mathbb{N}}$ is related to the space $\mathbb{N} \subseteq \mathbb{N}$ of partial functions $f: \subseteq \mathbb{N} \rightarrow \mathbb{N}$ that we can represent by $\delta_{\mathbb{N} \subseteq \mathbb{N}}\langle i, p\rangle:=\varphi_{i}^{p}$ for all $i \in \mathbb{N}, p \in \mathbb{N}^{\mathbb{N}}$. The exact relation between these two spaces is captured in the following lemma. The function $\iota$ essentially identifies $\uparrow$ (i.e., undefined) with $\perp$.

Lemma 7.1 (Space of partial functions). The function $\iota: \mathbb{N} \subseteq \mathbb{N} \rightarrow \overline{\mathbb{N}}^{\mathbb{N}}$ with

$$
\iota(p)(n):= \begin{cases}p(n) & \text { if } n \in \operatorname{dom}(p) \\ \perp & \text { otherwise }\end{cases}
$$

is computable and there is a computable function $\sigma: \overline{\mathbb{N}}^{\mathbb{N}} \rightarrow \mathbb{N} \subseteq \mathbb{N}$ such that $\sigma \circ \iota(p)$ is an extension of $p$ for all $p \in \mathbb{N} \subseteq \mathbb{N}$.

The proof is straightforward. We just note that a prefix of a name of $\perp$ can start like a name of a natural number $n \in \mathbb{N}$ and continue with dummy symbols 0 , which means that it is actually a name of $\perp$. Hence, we do not get that the spaces $\overline{\mathbb{N}}^{\mathbb{N}}$ and $\mathbb{N} \subseteq \mathbb{N}$ are computably isomorphic. However, Lemma 7.1 roughly speaking states that they are "isomorphic up to extensions". Hence, for every property that is invariant under extensions, it does not matter whether we work with $\overline{\mathbb{N}}^{\mathbb{N}}$ or $\mathbb{N} \subseteq \mathbb{N}$. This does, in particular, apply to stashing. Hence, for problems of type $f: \subseteq X \rightrightarrows \mathbb{N}$ we can also describe stashing by the following definition.

Definition 7.2. For every problem $f: \subseteq X \rightrightarrows \mathbb{N}$ we define ${ }^{\varphi} f: \subseteq X^{\mathbb{N}} \rightrightarrows \mathbb{N}^{\mathbb{N}}$ by

$$
\varphi^{\prime}\left(x_{n}\right)_{n \in \mathbb{N}}:=\left\{\langle i, p\rangle \in \mathbb{N}^{\mathbb{N}}:\left(\exists n \in \operatorname{dom}\left(\varphi_{i}^{p}\right)\right) \varphi_{i}^{p}(n) \in f\left(x_{n}\right)\right\}
$$

for all $\left(x_{n}\right)_{n \in \mathbb{N}} \in \operatorname{dom}(f)^{\mathbb{N}}$.

Hence, as an immediate corollary of Lemma 7.1 we obtain the following corollary.

Corollary 7.3 (Stashing for discrete outputs). $\Sigma f \equiv_{\mathrm{sW}}{ }^{\varphi} f$ for all problems $f: \subseteq X \rightrightarrows \mathbb{N}$. 
This again sheds light on the fact that stashing of parallelizable problems gives us computability-theoretic problems.

We note that the usage of the Gödel numbering and partial functions $\varphi_{i}^{p}$ in the previous sections was mostly related to the input side of problems. Hence, implicitly, we have worked with the input space $\mathbb{N} \subseteq \mathbb{N}$. Now, we want to get some better understanding of the effect of the space $\overline{\mathbb{N}}^{\mathbb{N}}$ on the output side. Corollary 7.3 can be seen as a way of replacing $\overline{\mathbb{N}}^{\mathbb{N}}$ on the output side by $\mathbb{N}^{\mathbb{N}}$.

For some problems, as those discussed in Propositions 5.2 and 5.10 it appeared that directly replacing $\overline{\mathbb{N}}^{\mathbb{N}}$ by $\mathbb{N}^{\mathbb{N}}$ on the output side was also possible without changing the degree. However, the problem NGEQ from Definition 6.1 is an example that shows that this is not always possible. If we just replace $\overline{\mathbb{N}}^{\mathbb{N}}$ by $\mathbb{N}^{\mathbb{N}}$ in $\Sigma(N G E Q)$, then we obtain NMAJ, which is not equivalent to $\Sigma$ (NGEQ) by Proposition 6.3.

Hence, it is useful to have upper bounds on the price that such a direct replacement of $\overline{\mathbb{N}}^{\mathbb{N}}$ by $\mathbb{N}^{\mathbb{N}}$ costs. This is exactly captured by the following retraction problem $\mathrm{RET}_{X}$ that we define together with the closely related extension problem EXT $\mathrm{E}_{X}$.

Definition 7.4 (Retraction and extension problems). For every represented space $X$ and $Y \subseteq \mathbb{N}$ we define the following problems:

(1) $\operatorname{RET}_{X}: \bar{X} \rightrightarrows X, x \mapsto\{y \in X: x \in X \Longrightarrow x=y\}$,

(2) $\mathrm{EXT}_{Y}: \subseteq \mathbb{N}^{\mathbb{N}} \rightrightarrows Y^{\mathbb{N}},\langle i, p\rangle \mapsto\left\{q \in Y^{\mathbb{N}}: q\right.$ is a total extension of $\left.\varphi_{i}^{p}\right\}$,

where $\operatorname{dom}\left(\operatorname{EXT}_{Y}\right):=\left\{\langle i, p\rangle: \operatorname{range}\left(\varphi_{i}^{p}\right) \subseteq Y\right\}$.

The parallelization $\widehat{\mathrm{RET}_{X}}$ captures the complexity of translating $\bar{X}^{\mathbb{N}}$ into $X^{\mathbb{N}}$. By Lemma 7.1 we obtain the following.

Corollary 7.5. $\widehat{\operatorname{RET}_{X}} \equiv_{\mathrm{sW}} \mathrm{EXT}_{X}$ for all $X \subseteq \mathbb{N}$.

The problem $\mathrm{RET}_{X}: \bar{X} \rightrightarrows X$ is a multi-valued retraction, i.e., a problem that satisfies $\left.\mathrm{RET}_{X}\right|_{X}=\mathrm{id}_{X}$. And it is the simplest such retraction in terms of strong Weihrauch reducibility. Hence, we obtain the following result.

Proposition 7.6 (Multi-retraceability). Let $X$ be a represented space. Then there exists a computable multi-valued retraction $R: \bar{X} \rightrightarrows X$ if and only if $\mathrm{RET}_{X}$ is computable.

Proof. It is clear that RET $X$ is a multi-valued retraction, which yields the "if"-direction of the proof. On the other hand, if there is a computable multi-valued retraction $R$, then $\operatorname{RET}_{X} \leq_{\mathrm{sW}} R$ and hence RET $X$ is computable too.

Spaces that allow for computable multi-valued retractions were called multi-retraceable in [BG20]. This condition was further studied by Hoyrup in [Hoy21] and related to fixed-point properties. Here we rather have to deal with spaces that are not multi-retraceable. In these cases we can consider the complexity of $\operatorname{RET}_{X}$ as a measure of how far the space $X$ is away from multi-retraceability or, in other words, how difficult it is to determine total extensions. We gain some upper bounds directly from [BG21, Proposition 2.10].

Fact 7.7. $\mathrm{RET}_{X} \leq_{\mathrm{sW}} \lim$ for all totally represented $X$ and $\mathrm{RET}_{\mathbb{N}} \leq_{\mathrm{sW}} \mathrm{C}_{\mathbb{N}}$.

For finite $X \subseteq \mathbb{N}$ we can classify $\mathrm{RET}_{X}$ somewhat more precisely. It is quite easy to see that the retraction problem $\mathrm{RET}_{n}$ is just equivalent to all-or-unique choice $\mathrm{AUC}_{n}$ and hence located in between LLPO $=\mathrm{AUC}_{2}$ and $\mathrm{C}_{n}$, as well as LPO. 
Proposition 7.8 (Retraction problem). LLPO $\leq_{\mathrm{sW}} \mathrm{RET}_{n} \equiv_{\mathrm{sW}} \mathrm{AUC}_{n} \leq_{\mathrm{sW}} \mathrm{C}_{n}$, and $\mathrm{RET}_{n} \leq_{\mathrm{sW}} \mathrm{RET}_{\mathbb{N}} \equiv_{\mathrm{W}} \mathrm{LPO}$ for all $n \geq 2$.

Proof. We start with proving $\mathrm{RET}_{n} \leq_{\mathrm{sW}} \mathrm{AUC}_{n}$. Given a name $p$ of $x \in \overline{\mathrm{N}}$ we generate a name $q$ of the set $\{0,1, \ldots, n-1\}$, as long as only dummy information appears in $p$. If some prefix of $p$ starts to look like a name of some $k<n$, then we modify the output $q$ to a name of the set $\{k\}$. Since $k \in \operatorname{RET}_{n}(x)$, this yields the desired reduction.

Now we consider the inverse reduction $\mathrm{AUC}_{n} \leq_{\mathrm{sW}} \mathrm{RET}_{n}$. Given a name $p$ of a set $A \subseteq\{0,1, \ldots, n-1\}$ with $A=\{0,1, \ldots, n-1\}$ or $A=\{k\}$ for some $k<n$, we generate a name of $\perp$ as long as the name looks like a name of $\{0,1, \ldots, n-1\}$. In the moment where the represented set is clearly smaller, we wait until the information suffices to identify the singleton $\{k\}$, and then we modify the output to an output of $k \in \overline{\{0,1, \ldots, n-1\}}$. If we apply $\operatorname{RET}_{n}$ to the generated point in $\overline{\{0,1, \ldots, n-1\}}$ it yields a point in $A$. This establishes the desired reduction.

The reductions $\mathrm{LLPO}=\mathrm{AUC}_{2} \leq_{\mathrm{sW}} \mathrm{AUC}_{n} \leq_{\mathrm{sW}} \mathrm{C}_{n}$ are clear for all $n \geq 2$. The reduction $\mathrm{RET}_{n} \leq_{\mathrm{sW}} \mathrm{RET}_{\mathbb{N}}$ is easy to see, as well as $\mathrm{RET}_{\mathbb{N}} \leq_{\mathrm{W}} \mathrm{LPO}$. For the latter reduction, we consider a name $p$ of an input $x \in \overline{\mathbb{N}}$. We use LPO in order to decide whether $p \neq \widehat{0}$. If $p=\widehat{0}$, then we can produce any output $k \in \mathbb{N}$; otherwise we search for the first non-zero component $k+1$ in $p$ and produce the corresponding $k$ as output. This yields the reduction $R E T_{\mathbb{N}} \leq_{W}$ LPO. Finally, the reduction LPO $\leq_{W} R T_{\mathbb{N}}$ can be seen as follows. Given an input $p \in \mathbb{N}^{\mathbb{N}}$, we seek the first non-zero component. If this component appears in position $n$, then we generate the output $x=n \in \overline{\mathbb{N}}$. If there is no non-zero component, then we generate the output $x=\perp \in \overline{\mathbb{N}}$. Now given some $k \in \operatorname{RET}_{\mathbb{N}}(x)$ and $p$, we search a non-zero component in $p$ up to position $k$. If such a non-zero component appears, then $\operatorname{LPO}(p)=0$; otherwise $\operatorname{LPO}(p)=1$. This describes the reduction $\mathrm{LPO} \leq_{\mathrm{W}} \mathrm{RET}_{\mathbb{N}}$.

Using this result, we obtain the pentagon of $\mathrm{RET}_{n}$ in the Weihrauch lattice. By Fact 1.10 we have $\widehat{\mathrm{LLPO}} \equiv_{\mathrm{sW}} \widehat{\mathrm{C}_{n}} \equiv_{\mathrm{sW}} \mathrm{WKL}$, which implies $\widehat{\mathrm{RET}_{n}} \equiv_{\mathrm{sW}} \mathrm{WKL}$. On the other hand, by Proposition 5.10 we have $\underline{\mathrm{LLPO}} \equiv_{\mathrm{sW}} \underline{\mathrm{LPO}} \equiv_{\mathrm{sW}}$ DIS and this yields $\mathrm{RET}_{n} \equiv_{\mathrm{W}}$ DIS.

Corollary 7.9 (Pentagon of retractions). RET $_{n} \equiv_{\mathrm{W}} \underline{\mathrm{RET}_{\mathbb{N}}} \equiv_{\mathrm{W}} \mathrm{DIS}, \widehat{\mathrm{RET}_{n}} \equiv_{\mathrm{sW}} \mathrm{WKL}$, and $\widehat{\mathrm{RET}_{\mathbb{N}}} \equiv_{\mathrm{W}} \lim$ for all $n \geq 2$.

This means that in the Weihrauch lattice the pentagon of $\mathrm{RET}_{n}$ for $n \geq 2$ looks like the pentagon of LLPO in Figure 4, while the pentagon of RET $_{\mathbb{N}}$ looks like the pentagon of LPO in Figure 5 (except that one cannot use strong Weihrauch reducibility). The first mentioned fact is also interesting, as the problems $\mathrm{RET}_{n}$ form a strictly increasing chain, as we prove next by a simple cardinality argument.

Proposition 7.10. $\mathrm{AUC}_{n}<_{\mathrm{W}} \mathrm{AUC}_{n+1}<_{\mathrm{W}} \mathrm{AUC}_{\mathbb{N}}$ for all $n \geq 2$.

Proof. It suffices to prove $\mathrm{AUC}_{n+1} \mathbb{Z}_{\mathrm{W}} \mathrm{AUC}_{n}$ for all $n \geq 2$. Let us assume for a contradiction that $\mathrm{AUC}_{n+1} \leq_{\mathrm{W}} \mathrm{AUC}_{n}$ for some $n \geq 2$ via computable $H, K$. Now consider the name $p=000 \ldots$ of the empty set $\emptyset=\operatorname{range}(p-1)$. Then $a_{k}:=H\langle p, k\rangle \in\{0, \ldots, n\}$ for $k<n$ satisfy $\left|\left\{a_{0}, \ldots, a_{n-1}\right\}\right| \leq n$. For each $k<n$ there is some prefix of $p$ of length $l_{k}$ that suffices to produce the output $a_{k}$. For the prefix $l:=\max \left\{l_{0}, \ldots, l_{n}\right\}$ we have

$$
\left|H\left\langle p \mid{ }_{l} \mathbb{N}^{\mathbb{N}} \times\{0, \ldots, n\}\right\rangle\right|=\left|\left\{a_{0}, \ldots, a_{n-1}\right\}\right| \leq n,
$$

despite the fact that $\left.p\right|_{l} \mathbb{N}^{\mathbb{N}}$ contains names of all singleton sets $A \subseteq\{0,1, \ldots, n\}$. Since there are $n+1$ such sets, this is a contradiction! 
That means that with the problems $\mathrm{RET}_{n} \equiv_{\mathrm{sW}} \mathrm{AUC}_{n}$ we have a strictly increasing sequence of problems in between LLPO and LPO that all parallelize and stash to the same problems, respectively. This is a strong refutation of injectivity of the following operation.

Corollary 7.11. The map $f \mapsto(\underset{\sim}{f} \widehat{f})$ is not injective on Weihrauch degrees.

This means that pentagons do not characterize the Weihrauch degrees that generate them, even though they reveal some useful information about them.

\section{Limit Avoidance and a Phase Transition}

The pentagons determined so far are all related to the discontinuity problem. This could lead to the false conclusion that DIS is the bottom vertex of typical pentagons. However, this is rather based on the fact that our study of pentagons was motivated by an analysis of the discontinuity problem. In this closing section we study the weakest problem known to us whose stashing is not DIS. This is the limit avoidance problem $\mathrm{NLIM}_{\mathbb{N}}$, defined below. We define it together with the related non-lowness problem NLOW.

Definition 8.1 (Problems related to limits). We consider the following problems for $X \subseteq \mathbb{N}$ :

(1) $\operatorname{NLIM}_{X}: \subseteq X^{\mathbb{N}} \rightrightarrows X, p \mapsto\left\{k \in X: \lim _{n \rightarrow \infty} p(n) \neq k\right\}$ with $\operatorname{dom}\left(\mathrm{NLIM}_{X}\right)=\left\{p:(p(n))_{n \in \mathbb{N}}\right.$ converges $\}$,

(2) NLOW : D $\rightrightarrows \mathcal{D}, a \mapsto\left\{b \in \mathcal{D}: b^{\prime} \not \leq a^{\prime}\right\}$.

The following fact is easy to see:

Fact 8.2. $A C C_{\mathbb{N}} \leq_{\mathrm{sW}} \mathrm{NLIM}_{\mathbb{N}} \leq_{\mathrm{sW}} \mathrm{NLIM}_{2} \equiv_{\mathrm{sW}} \lim _{2} \leq_{\mathrm{sW}} \lim _{\mathbb{N}} \equiv_{\mathrm{sW}} C_{\mathbb{N}}$.

If we can prove that DIS $<_{W} \mathrm{NLIM}_{\mathbb{N}}$ holds, then this implies that the stashings of all problems above $\mathrm{NLIM}_{\mathbb{N}}$ also lie above DIS. We prove the following stronger result.

Proposition 8.3. NLIM $_{\mathbb{N}} Z_{W} W K L$.

Proof. We recall that we are working with

$$
\underline{\mathrm{NLIM}_{\mathbb{N}}}: \subseteq \mathbb{N}^{\mathbb{N}} \rightrightarrows \overline{\mathbb{N}}^{\mathbb{N}},\left\langle p_{0}, p_{1}, p_{2}, \ldots\right\rangle \mapsto\left\{q \in \overline{\mathbb{N}}^{\mathbb{N}}:(\exists n) \lim _{i \rightarrow \infty} p_{i}(n) \neq q(n) \in \mathbb{N}\right\} .
$$

Let us assume for a contradiction that $\mathrm{NLIM}_{\mathbb{N}} \leq_{W} \mathrm{WKL}$ holds. As WKL is a cylinder (i.e., satisfies id $\times W K L \equiv_{s W} W K L$ ), it follows that NLIM $_{\mathbb{N}} \leq_{s W} W K L$. Since stashing is an interior operator, this yields $\underline{N L I M}_{\mathbb{N}} \leq_{s W} W_{K L}{ }^{\mathcal{D}} \equiv_{s W}$ PA by Fact 5.7. But this means that every fixed $r \in 2^{\mathbb{N}}$ of PA-degree has the property that it computes some fixed $q$ with $q \in \mathrm{NLIM}_{\mathbb{N}}\left\langle p_{0}, p_{1}, p_{2}, \ldots\right\rangle$ for all computable $\left\langle p_{0}, p_{1}, p_{2}, \ldots\right\rangle \in \operatorname{dom}\left(\mathrm{NLIM}_{\mathbb{N}}\right)$. This is because the reduction $\mathrm{NLIM}_{\mathbb{N}} \leq_{\mathrm{sW}} \mathrm{PA}$ is a strong one and hence $q$ only depends on $r$. There are low $r \in 2^{\mathbb{N}}$ of PA-degree, i.e., such that $r^{\prime} \leq_{\mathrm{T}} \emptyset^{\prime}$. This is because the set of diagonally non-computable binary functions is co-c.e. closed, every such function is of PA-degree (see [Soa16, Theorem 10.3.3]) and by the low basis theorem there is a low function among those (see [Soa16, Theorem 3.7.2]). Hence there is some fixed low $q$ with $q \in \underline{\mathrm{NLIM}_{\mathbb{N}}}\left\langle p_{0}, p_{1}, p_{2}, \ldots\right\rangle$ for all computable $\left\langle p_{0}, p_{1}, p_{2}, \ldots\right\rangle \in \operatorname{dom}\left(\mathrm{NLIM}_{\mathbb{N}}\right)$. By Corollary 7.9 this implies that there is some $t \in \widehat{\operatorname{RET}_{\mathbb{N}}}(q)$, which is limit computable, as $\widehat{\operatorname{RET}_{\mathbb{N}}} \leq_{\mathrm{sW}}$ lim and limit computable problems yield some limit computable outputs on low inputs. This means that for a fixed limit computable $t: \mathbb{N} \rightarrow \mathbb{N}$ we have that $\lim _{i \rightarrow \infty} p_{i} \neq t$ for all computable $\left\langle p_{0}, p_{1}, p_{2}, \ldots\right\rangle \in \operatorname{dom}\left(\underline{\mathrm{NLIM}}_{\mathbb{N}}\right)$, which is impossible, as $\lim _{i \rightarrow \infty} p_{i}$ is limit computable. 
We mention that Fact 8.2 implies that $\mathrm{DNC}_{\mathbb{N}} \leq_{\mathrm{sW}} \widehat{\mathrm{NLIM}_{\mathbb{N}}} \leq_{\mathrm{sW}}$ lim. We leave it to a future study to determine the exact pentagons of the problems listed in Fact 8.2 other than $\mathrm{ACC}_{\mathbb{N}}$. Here we just mention one upper bound on $\Pi \Sigma\left(\mathrm{NLIM}_{\mathbb{N}}\right)$.

Since DIS $<_{W}$ NON $<_{W}$ WKL it is clear that Proposition 8.3 implies NLIM $_{\mathbb{N}} \not_{W}$ DIS and also $\Pi \Sigma\left(N_{L} I M_{\mathbb{N}}\right) \not Z_{W} N O N$. It is not difficult to show that the non-lowness problem is an upper bound for $\Pi \Sigma\left(\mathrm{NLIM}_{\mathbb{N}}\right)$.

Proposition 8.4. $\Pi \Sigma\left(\mathrm{NLIM}_{\mathbb{N}}\right) \leq_{\mathrm{sW}}$ NLOW.

Proof. For simplicity we work with the equivalent definition

$$
\text { NLOW }: \mathbb{N}^{\mathbb{N}} \rightrightarrows \mathbb{N}^{\mathbb{N}}, p \mapsto\left\{q \in \mathbb{N}^{\mathbb{N}}: q^{\prime} \not \mathbb{\mathrm { T }}_{\mathrm{T}} p^{\prime}\right\} \text {. }
$$

We first note that NLOW is parallelizable: this follows since $q^{\prime} \mathbb{Z}_{\mathrm{T}}\left\langle p_{0}, p_{1}, p_{2}, \ldots\right\rangle^{\prime}$ implies $q^{\prime} \not_{\mathrm{T}} p_{i}^{\prime}$ for all $i \in \mathbb{N}$. Hence, it suffices to show $\mathrm{NLIM}_{\mathbb{N}} \leq_{\mathrm{sW}} \mathrm{NLOW}$ in order to obtain $\Pi \Sigma\left(\mathrm{NLIM}_{\mathbb{N}}\right) \leq_{\mathrm{sW}}$ NLOW. Now given $p:=\left\langle p_{0}, p_{1}, p_{2}, \ldots\right\rangle$ such that $r=\lim _{i \rightarrow \infty} p_{i}$ exists in $\mathbb{N}^{\mathbb{N}}$, we have $r \leq_{\mathrm{T}} p^{\prime}$. Now let $q \in \mathbb{N}^{\mathbb{N}}$ be such that $q^{\prime} \mathbb{Z}_{\mathrm{T}} p^{\prime}$. Then using the time complexity function $\Phi_{i}^{q}$ we can define by $s\langle i, n\rangle:=\Phi_{i}^{q}(n)$ a partial function $s: \subseteq \mathbb{N} \rightarrow \mathbb{N}$ that is computable from $q$. This function has the property that every total extension $t: \mathbb{N} \rightarrow \mathbb{N}$ of it computes $q^{\prime}$. This is because we can simulate the computation of $\varphi_{i}^{q}(n)$ for given $i, n$ for $t\langle i, n\rangle$ time steps. And either the computation halts within this time bound, which implies $q^{\prime}\langle i, n\rangle=1$ or it does not halt, which implies that $t\langle i, n\rangle$ is not a correct time bound, hence $\Phi_{i}^{q}(n)$ is undefined and hence $q^{\prime}\langle i, n\rangle=0$. By Lemma 7.1 we can consider $s$ as a function of type $s: \overline{\mathbb{N}} \rightarrow \mathbb{N}$. Since every total extension $t: \mathbb{N} \rightarrow \mathbb{N}$ of it computes $q^{\prime}$, we obtain $t \mathbb{K}_{\mathrm{T}} p^{\prime}$, which implies $r \neq t$ for every such extension $t$. Hence there is some $n \in \mathbb{N}$ with $r(n) \neq s(n) \in \mathbb{N}$, i.e., $s \in \underline{\operatorname{NLIM}_{\mathbb{N}}}(p)$. This establishes the reduction $\Pi \Sigma\left(\mathrm{NLIM}_{\mathbb{N}}\right) \leq_{\mathrm{sW}} \mathrm{NLOW}$.

\section{Conclusions}

We have introduced the stashing operation as a dual of parallelization and we have proved that it is an interior operator. The action of parallelization and stashing on Weihrauch degrees naturally leads to the study of stashing-parallelization pentagons, which can be used to describe a number of natural Weihrauch degrees. In many cases of the studied pentagons the discontinuity problem featured as the bottom problem of the respective pentagon. However, we were also able to identify a phase transition point, where this no longer happens to be the case. The duality inherent in pentagons needs to be studied further in order to simplify the calculation of pentagons, which tends to be difficult in more advanced examples.

\section{REFERENCES}

[BdBP12] Vasco Brattka, Matthew de Brecht, and Arno Pauly. Closed choice and a uniform low basis theorem. Annals of Pure and Applied Logic, 163:986-1008, 2012.

[BG11a] Vasco Brattka and Guido Gherardi. Effective choice and boundedness principles in computable analysis. The Bulletin of Symbolic Logic, 17(1):73-117, 2011.

[BG11b] Vasco Brattka and Guido Gherardi. Weihrauch degrees, omniscience principles and weak computability. The Journal of Symbolic Logic, 76(1):143-176, 2011.

[BG20] Vasco Brattka and Guido Gherardi. Weihrauch goes Brouwerian. The Journal of Symbolic Logic, 85(4):1614-1653, 2020. 
[BG21] Vasco Brattka and Guido Gherardi. Completion of choice. Annals of Pure and Applied Logic, 172(3):102914, 2021.

[BGH15] Vasco Brattka, Guido Gherardi, and Rupert Hölzl. Probabilistic computability and choice. Information and Computation, 242:249-286, 2015.

[BGM12] Vasco Brattka, Guido Gherardi, and Alberto Marcone. The Bolzano-Weierstrass theorem is the jump of weak König's lemma. Annals of Pure and Applied Logic, 163:623-655, 2012.

[BGP21] Vasco Brattka, Guido Gherardi, and Arno Pauly. Weihrauch complexity in computable analysis. In Vasco Brattka and Peter Hertling, editors, Handbook of Computability and Complexity in Analysis, Theory and Applications of Computability, pages 367-417. Springer, Cham, 2021.

[BHK17] Vasco Brattka, Matthew Hendtlass, and Alexander P. Kreuzer. On the uniform computational content of computability theory. Theory of Computing Systems, 61(4):1376-1426, 2017.

[BHK18] Vasco Brattka, Matthew Hendtlass, and Alexander P. Kreuzer. On the uniform computational content of the Baire category theorem. Notre Dame Journal of Formal Logic, 59(4):605-636, 2018.

[BP18] Vasco Brattka and Arno Pauly. On the algebraic structure of Weihrauch degrees. Logical Methods in Computer Science, 14(4:4):1-36, 2018.

[Bra99] Vasco Brattka. Computable invariance. Theoretical Computer Science, 210:3-20, 1999.

[Bra05] Vasco Brattka. Effective Borel measurability and reducibility of functions. Mathematical Logic Quarterly, 51(1):19-44, 2005.

[Bra20] Vasco Brattka. The discontinuity problem. arXiv 2012.02143, 2020.

[Dzh19] Damir D. Dzhafarov. Joins in the strong Weihrauch degrees. Mathematical Research Letters, 26(3):749-767, 2019.

[HK14a] Kojiro Higuchi and Takayuki Kihara. Inside the Muchnik degrees I: Discontinuity, learnability and constructivism. Annals of Pure and Applied Logic, 165(5):1058-1114, 2014.

[HK14b] Kojiro Higuchi and Takayuki Kihara. Inside the Muchnik degrees II: The degree structures induced by the arithmetical hierarchy of countably continuous functions. Annals of Pure and Applied Logic, 165(6):1201-1241, 2014.

[Hoy21] Mathieu Hoyrup. The fixed-point property for represented spaces. hal-03117745, 2021.

[HRSZ17] Rupert Hölzl, Dilip Raghavan, Frank Stephan, and Jing Zhang. Weakly represented families in reverse mathematics. In Adam Day, Michael Fellows, Noam Greenberg, Bakhadyr Khoussainov, Alexander Melnikov, and Frances Rosamond, editors, Computability and Complexity: Essays Dedicated to Rodney G. Downey on the Occasion of His 60th Birthday, volume 10010 of Lecture Notes in Computer Science, pages 160-187. Springer, Cham, 2017.

[Joc89] Carl G. Jockusch, Jr. Degrees of functions with no fixed points. In Logic, methodology and philosophy of science, VIII (Moscow, 1987), volume 126 of Stud. Logic Found. Math., pages 191-201, Amsterdam, 1989. North-Holland.

[KP16] Takayuki Kihara and Arno Pauly. Dividing by zero-how bad is it, really? In 41st International Symposium on Mathematical Foundations of Computer Science, volume 58 of LIPIcs. Leibniz Int. Proc. Inform., pages Art. No. 58, 14. Schloss Dagstuhl. Leibniz-Zent. Inform., Wadern, 2016.

[Mil04] Joseph S. Miller. Degrees of unsolvability of continuous functions. The Journal of Symbolic Logic, 69(2):555-584, 2004.

[MM68] Webb Miller and D. A. Martin. The degrees of hyperimmune sets. Zeitschrift für Mathematische Logik und Grundlagen der Mathematik, 14:159-166, 1968.

[Myl92] Uwe Mylatz. Vergleich unstetiger Funktionen in der Analysis. PhD thesis, Fachbereich Informatik, FernUniversität Hagen, 1992. Diplomarbeit.

[Myl06] Uwe Mylatz. Vergleich unstetiger Funktionen: "Principle of Omniscience" und Vollständigkeit in der $C$-Hierarchie. PhD thesis, Faculty for Mathematics and Computer Science, University Hagen, Hagen, Germany, 2006. Ph.D. thesis.

[NP19] Hugo Nobrega and Arno Pauly. Game characterizations and lower cones in the Weihrauch degrees. Logical Methods in Computer Science, 15(3):Paper No. 11, 29, 2019.

[Pau11] Arno Pauly. Computable Metamathematics and its Application to Game Theory. PhD thesis, University of Cambridge, Computer Laboratory, Clare College, Cambridge, 2011. Ph.D. thesis.

[Sim77] Stephen G. Simpson. Degrees of unsolvability: A survey of results. In Jon Barwise, editor, Handbook of Mathematical Logic, volume 90 of Studies in Logic and the Foundations of Mathematics, pages 631-652. North-Holland, Amsterdam, 1977. 
[Soa16] Robert I. Soare. Turing Computability. Theory and Applications of Computability. Springer, Berlin, Heidelberg, 2016.

[vS89] Thorsten von Stein. Vergleich nicht konstruktiv lösbarer Probleme in der Analysis. PhD thesis, Fachbereich Informatik, FernUniversität Hagen, 1989. Diplomarbeit.

[Wei92] Klaus Weihrauch. The TTE-interpretation of three hierarchies of omniscience principles. Informatik Berichte 130, FernUniversität Hagen, Hagen, September 1992.

[Wei00] Klaus Weihrauch. Computable Analysis. Springer, Berlin, 2000.

\section{ACKNOWLEDGMENTS}

This work has been supported by the National Research Foundation of South Africa (Grant Number 115269). 